\title{
QUASI-DIAGONAL FLOWS II
}

\author{
A. KISHIMOTO
}

\begin{abstract}
Two similar notions defined for flows, quasi-diagonality and pseudo-diagonality, are shown to be equivalent; so approximately inner flows on a quasi-diagonal $C^{*}$-algebra are quasi-diagonal (not just pseudo-diagonal). We define a notion of MF flow which is weaker than quasi-diagonality and study equivalent conditions following Blackadar and Kirchberg's results on MF algebras and we characterize the dual flow of such on the crossed product as a dual MF flow. In the same spirit we introduce a notion of NF flow and show that NF flows are MF flows on nuclear $C^{*}$ algebras, or equivalently, quasi-diagonal flows on nuclear $C^{*}$-algebras. We also introduce a notion of strong quasi-diagonality (in parallel with strong quasi-diagonality versus quasi-diagonality for $C^{*}$-algebras), whose examples contain AF flows.
\end{abstract}

\section{Introduction}

We mean by a flow a strongly continuous one-parameter automorphism group of a $C^{*}$-algebra. We refer to [4], [14] for some background on flows. We are particularly interested in approximately inner flows since they have close relevance to applications to physics and were a cause for $C^{*}$-algebras to have been introduced. But we are still trying to understand the situations surrounding approximately inner flows (see, e.g., [3], [8]).

We have defined two similar notions for flows on $C^{*}$-algebras: pseudodiagonality and quasi-diagonality, in [11], which are naturally derived from the notion of quasi-diagonality for $C^{*}$-algebras (e.g., [15], [16]). But as we shall see in this note they are in fact equivalent. Thus quasi-diagonality holds for approximately inner flows on quasi-diagonal $C^{*}$-algebras. For example if $\alpha$ is an approximately inner flow on an AF algebra $A$ then there is a covariant representation $(\pi, U)$ of $(A, \alpha)$ such that $\pi$ is faithful and $(\pi(A), U)$ is quasidiagonal, i.e., $\left\|\left[E_{n}, \pi(x)\right]\right\| \rightarrow 0$ for $x \in A$ and $\sup \left\{\left\|\left[E_{n}, U_{t}\right]\right\| \mid-1 \leq\right.$ $t \leq 1\} \rightarrow 0$ for some increasing sequence $\left(E_{n}\right)$ of finite-rank projections on $\mathscr{H}_{\pi}$ with $\lim _{n} E_{n}=1$. Note also that for any covariant representation $(\pi, U)$ there is an increasing sequence $\left(E_{n}\right)$ of finite-rank projections on $\mathscr{H}_{\pi}$ with $\lim _{n} E_{n}=1$ and a sequence $\left(V_{n}\right)$ of unitary flows such that $V_{n, t} E_{n}=V_{n, t}$ and $\left\|\left[E_{n}, \pi(x)\right]\right\| \rightarrow 0$ and $\left\|E_{n} \pi \alpha_{t}(x) E_{n}-V_{n, t} E_{n} \pi(x) E_{n} V_{n, t}^{*}\right\| \rightarrow 0$ uniformly in $t$ on every compact subset of $\mathrm{R}$ for any $x \in A$. If a covariant representation

Received 25 October 2010. 
$(\pi, U)$ induces a faithful representation of the crossed product then $(\pi(A), U)$ is quasi-diagonal by Voiculescu's theorem (Theorem 3.1 of [11]). Thus an approximately inner flow on an AF algebra can be approximated by flows on finite-dimensional $C^{*}$-algebras in a sense.

We have also noted in [11] we could define a notion of MF flows when the $C^{*}$-algebra is separable, which is derived from pseudo-diagonality, following the notion of MF algebras introduced and studied by Blackadar and Kirchberg [1]. We will examine this notion closely following [1]. See Theorem 3.10 for equivalent conditions.

Let us be specific about the definition of MF flows. Let $M_{n}$ be the $C^{*}$-algebra of $n \times n$ matrices. Any flow on $M_{n}$ is given as $t \mapsto \operatorname{Ad} e^{i t h}$ with $h=h^{*} \in M_{n}$. Let $\left(k_{n}\right)$ be a sequence of natural numbers and let $B=\prod_{n=1}^{\infty} M_{k_{n}}$ be the $C^{*}$ algebra consisting of bounded sequences ( $\left.x_{n}\right)$ with $x_{n} \in M_{k_{n}}$. Let $\beta_{n}$ be a flow on $M_{k_{n}}$ and let $\beta_{t}=\prod \beta_{n, t}, t \in \mathrm{R}$ as automorphisms of $B$. Since $t \mapsto \beta_{t}$ is not continuous on $B$ in general, we let $B_{\beta}$ be the maximal $C^{*}$-subalgebra of $B$ on which $t \mapsto \beta_{t}$ is continuous. Thus $\beta$ restricts to a flow on $B_{\beta}$. Let $I=\bigoplus_{n=1}^{\infty} M_{k_{n}}$ be the $C^{*}$-algebra consisting of sequences converging to zero, which is an ideal of $B$ contained in $B_{\beta}$ and is left invariant under $\beta$. We denote by the same symbol $\beta$ the flow on $B_{\beta} / I$ induced from $\beta$ on $B_{\beta}$.

When $\alpha$ is a flow on a separable $C^{*}$-algebra $A$ we consider the following conditions:

(1) There is an isomorphism $\phi$ of $A$ into $B_{\beta} / I$ such that $\phi \alpha_{t}=\beta_{t} \phi$ (for some $B=\prod_{n=1}^{\infty} M_{k_{n}}$ and $\left.\beta=\prod_{n=1}^{\infty} \beta_{n}\right)$.

(2) There is a completely positive (CP) contraction $\phi$ of $A$ into $B_{\beta}$ such that $Q \phi$ is an isomorphism and $Q \phi \alpha_{t}=\beta_{t} Q \phi$, where $Q$ is the quotient map of $B_{\beta}$ onto $B_{\beta} / I$.

(3) There is an isomorphism $\phi$ of $A$ into $B_{\beta}$ such that $\phi \alpha_{t}=\beta_{t} \phi$.

We will call $\alpha$ an MF flow if it satisfies the first condition. The second condition on $\alpha$ is equivalent to $\alpha$ 's being quasi-diagonal (by Theorem 2.3), which is stronger than the first in general (if $A$ is not nuclear). We will call $\alpha$ an $R F$ flow if it satisfies the third condition. In this case $A$ is residually finite-dimensional as a $C^{*}$-algebra. This is stronger than the second because, if $Q \phi$ is not an injection or $\phi(A) \cap I$ is non-zero, there is another $(B, \beta)$, where $B$ may be obtained by repeating an infinite copies of each $M_{k_{n}}$ from the original $B$, and an isomorphism $\psi$ of $A$ into this new $B_{\beta}$ such that $Q \psi$ is an isomorphism and $Q \psi \alpha_{t}=\beta_{t} Q \psi$. We note in 3.5 that we may replace all $\left(M_{k_{n}}, \beta_{n}\right)$ by a single $(\mathscr{K}, \operatorname{Ad} \lambda)$ in the definition of MF flows, where $\mathscr{K}$ is the $C^{*}$-algebra of compact operators on $L^{2}(\mathrm{R})$ and $\lambda$ is the unitary flow defined by $\left(\lambda_{t} \xi\right)(s)=\xi(s-t)$. We also note in 3.12 that an MF flow is obtained as a quotient of an RF 
flow. As in the case of pseudo-diagonal flows, if $\alpha$ is an MF flow on a unital $C^{*}$-algebra, it has KMS states for all inverse temperatures as shown in 3.14. This is what motivates us to introduce MF flows. We shall also introduce a notion of dual MF flows; $\alpha$ is a dual MF flow on $A$ if $(A, \alpha)$ is realized in $\left(\left(\prod M_{k_{n}} \otimes C_{0}(\mathrm{R})\right)_{\gamma} / \bigoplus M_{k_{n}} \otimes C_{0}(\mathrm{R}), \gamma\right)$ for some $\left(k_{n}\right)$ where $\gamma=\prod \gamma_{n}$ and $\gamma_{n}$ is the flow induced from translations on R. It follows in 3.19 that $\alpha$ is an MF flow (resp. a dual MF flow) if and only if $\hat{\alpha}$ is a dual MF flow (resp. a MF flow) on the crossed product $A \times{ }_{\alpha}$ R.

We will also define a notion of $N F$ flows following [1] and study some equivalent conditions in Theorem 4.7. It will turn out that an NF flow is an MF flow on a nuclear $C^{*}$-algebra as expected and has a characterization in terms of $\mathrm{CP}$ contractions through finite-dimensional $C^{*}$-algebras as follows: There is a sequence of flows $\left(B_{n}, \beta_{n}\right)$ with $B_{n}$ finite-dimensional and $\mathrm{CP}$ contractions $\sigma_{n}: A \rightarrow B_{n}$ and $\tau_{n}: B_{n} \rightarrow A$ such that $\tau_{n} \sigma_{n} \rightarrow$ id, $\left\|\sigma_{n}(x y)-\sigma_{n}(x) \sigma_{n}(y)\right\| \rightarrow 0$ for all $x, y \in A$, and $\left\|\sigma_{n} \alpha_{t}-\beta_{n, t} \sigma_{n}\right\| \rightarrow 0$ uniformly in $t$ on every compact subset of R. By the way quasi-diagonality is characterized without $\tau_{n}$ in the above condition replacing $\tau_{n} \sigma_{n} \rightarrow$ id by $\left\|\sigma_{n}(x)\right\| \rightarrow\|x\|, x \in A$ (see Theorem 1.5 of [11]).

We will also define a notion of strongly quasi-diagonal flows, which is naturally stronger than quasi-diagonality, and note that such a flow on a separable $C^{*}$-algebra is obtained as the limit of a canonical increasing sequence of RF flows after a cocycle perturbation (see 5.9 for details). An AF flow is strongly quasi-diagonal (see 5.6), where an AF flow is defined as the limit of an increasing sequence of FD flows (i.e., flows on finite-dimensional algebras).

I would like to express my gratitude to Ola Bratteli for inviting me to Oslo in October 2010 and giving me an opportunity to present a seminar talk on the topic dealt in this note. This was undoubtedly inspired by long-term collaboration with him and another co-author Derek Robinson who was in the audience. I would also like to record my gratitude to all the participants.

\section{Quasi-diagonal flows}

First we note the following result, which we should have noticed before.

Proposition 2.1. Pseudo-digonality and quasi-diagonaliy for flows are equivalent.

Proof. We shall show that the condition (2) of Theorem 1.6 of [11] implies the condition (2) of Theorem 1.5 of [11]. The converse is trivial.

Let $\alpha$ be a pseudo-diagonal flow on $A$. Hence $\alpha$ satisfies the following condition: For any finite subset $\mathscr{F}$ of $A, T>0$, and $\delta>0$ there is a finitedimensional $C^{*}$-algebra $B$, a flow $\beta$ on $B$ and a CP map $\phi$ of $A$ into $B$ such 
that $\|\phi\| \leq 1,\|\phi(x)\| \geq(1-\delta)\|x\|$ for $x \in \mathscr{F}$ and $\|\phi(x) \phi(y)-\phi(x y)\| \leq$ $\delta\|x\|\|y\|$ for $x, y \in \mathscr{F}$, and $\left\|\beta_{t} \phi(x)-\phi \alpha_{t}(x)\right\| \leq \epsilon\|x\|$ for $x \in \mathscr{F}$ and $t \in[-T, T]$. This is slightly different from the condition (2) of 1.6 of [11] but they are equivalent as we can see easily. Especially we have allowed $T$ to be arbitrarily large instead of fixing it to be 1 .

Let $\epsilon>0$ be smaller than 1 . We define a CP map $\psi$ of $A$ into $B$ by

$$
\psi=\frac{\epsilon}{2} \int e^{-\epsilon|t|} \beta_{-t} \phi \alpha_{t} d t
$$

For $x \in \mathscr{F}$ we compute

$$
\begin{aligned}
\|\psi(x)-\phi(x)\| & \leq \frac{\epsilon}{2} \int e^{-\epsilon|t|}\left\|\beta_{-t} \phi \alpha_{t}(x)-\phi(x)\right\| d t \\
& \leq \frac{\epsilon}{2} \int_{-T}^{T} e^{-\epsilon|t|} \delta\|x\| d t+\epsilon\|x\| \int_{|t| \geq T} e^{-\epsilon|t|} d t \\
& \leq\left(\delta+2 e^{-\epsilon T}\right)\|x\| .
\end{aligned}
$$

Thus if we set $\delta=\epsilon / 2$ and $T=\epsilon^{-1} \log (4 / \epsilon)$, we obtain that $\|\psi(x)-\phi(x)\| \leq$ $\epsilon\|x\|, x \in \mathscr{F}$. Hence we have that $\|\psi(x)\| \geq(1-2 \epsilon)\|x\|$ for $x \in \mathscr{F}$ and $\|\psi(x) \psi(y)-\psi(x y)\| \leq 3 \epsilon\|x\|\|y\|+\|\phi(x) \phi(y)-\phi(x y)\| \leq 4 \epsilon\|x\|\|y\|$ for $x, y \in \mathscr{F}$.

Since

$$
\beta_{-t} \psi \alpha_{t}=\frac{\epsilon}{2} \int e^{-\epsilon|s-t|} \beta_{-s} \phi \alpha_{s} d s
$$

and $|s| \leq|s-t|+|t|$ and $|s-t| \leq|s|+|t|$, we obtain that $\left\|\beta_{-t} \psi \alpha_{t}-\psi\right\| \leq$ $e^{\epsilon|t|}-1$ or $\left\|\beta_{t} \psi-\psi \alpha_{t}\right\| \leq e^{\epsilon}-1$ for $t \in[-1,1]$. Thus the condition (2) of Theorem 1.5 of [11] is satisfied with $\psi$ in place of $\phi$ starting with a smaller $\epsilon$.

Remark 2.2. Suppose that $\alpha$ is an approximately inner flow on a quasidiagonal $C^{*}$-algebra $A$. Then $(\pi(A), U)$ is pseudo-diagonal for any covariant representation $(\pi, U)$ of $(A, \alpha)$ (see the proof of Proposition 2.17 of [11]). It follows from the above proof that for any covariant representation $(\pi, U)$ there is a covariant representation $(\rho, W)$ such that $\operatorname{Ker} \rho=\operatorname{Ker} \pi$ and $(\rho(A), W)$ is quasi-diagonal.

Theorem 2.3. Let $\alpha$ be a flow on a separable $C^{*}$-algebra A. Then the following conditions are equivalent.

(1) $\alpha$ is quasi-diagonal.

(2) $\alpha$ is pseudo-diagonal.

(3) There is a CP contraction $\phi$ of A into $\left(\prod_{n=1}^{\infty} M_{k_{n}}\right)_{\beta}$ such that $Q \phi$ is an isomorphism and $Q \phi \alpha_{t}=\beta_{t} Q \phi$ with $\beta=\prod_{n=1}^{\infty} \beta_{n}$ for some 
$\left(k_{n}\right)$ and some $\left(\beta_{n}\right)$, where $Q$ is the quotient map of $\left(\prod M_{k_{n}}\right)_{\beta}$ onto $\left(\prod M_{k_{n}}\right)_{\beta} / \bigoplus M_{k_{n}}$.

Proof. We have already shown that the first two conditions are equivalent.

Suppose $\alpha$ is pseudo-diagonal. Let $\left(x_{n}\right)$ be a dense sequence in $A$. We choose $M_{k_{n}}$ and a flow $\beta_{n}$ on $M_{k_{n}}$ and a CP contraction $\phi_{n}: A \rightarrow M_{k_{n}}$ such that $\left\|\phi_{n}\left(x_{k}\right)\right\| \geq(1-1 / n)\left\|x_{k}\right\|,\left\|\phi_{n}\left(x_{k}\right) \phi_{n}\left(x_{\ell}\right)-\phi\left(x_{k} x_{\ell}\right)\right\| \leq 1 / n$, and $\left\|\beta_{n, t} \phi\left(x_{k}\right)-\phi_{n} \alpha_{t}\left(x_{k}\right)\right\|<1 / n, t \in[-1,1]$ for all $k, \ell \leq n$. (As easily shown we may assume the target algebra for $\phi_{n}$ is a full matrix algebra.) We define a CP contraction $\phi$ of $A$ into $\prod_{n=1}^{\infty} M_{k_{n}}$ by $\phi(x)=\left(\phi_{n}(x)\right)_{n}$. Then one can show that $Q \phi$ is an isomorphism and $Q \phi \alpha_{t}=\beta_{t} Q \phi$. One can also show that $t \mapsto \beta_{t} \phi(x)=\left(\beta_{n, t} \phi_{n}(x)\right)_{n}$ is continuous since $\beta_{t} \phi(x)-\phi \alpha_{t}(x) \in \bigoplus M_{k_{n}}$. That is, we have that $\phi(A) \subset\left(\prod M_{k_{n}}\right)_{\beta}$.

Suppose (3). Let $\phi$ be a CP contraction of $A$ into $\left(\prod M_{k_{n}}\right)_{\beta}$ as given there. Let $\phi_{n}$ denote the component of $\phi$ mapping $A$ into $M_{k_{n}}$. Then for any finite subset $\mathscr{F}$ of $A \backslash\{0\}$ and $\epsilon>0$ there is an $n \in \mathrm{N}$ such that for $\phi^{(n)}=\prod_{k=n}^{\infty} \phi_{k}$ the conditions $\left\|\phi^{(n)}(x) \phi^{(n)}(y)-\phi^{(n)}(x y)\right\| \leq \epsilon\|x\|\|y\|$, and $\| \beta_{t} \phi^{(n)}(x)-$ $\phi^{(n)} \alpha_{t}(x)\|\leq \epsilon\| x \|, t \in[-1,1]$ are satisfied for all $x, y \in \mathscr{F}$. We then find $m>n$ such that $\prod_{k=n}^{m} \phi_{k}$ instead of $\phi^{(n)}$ still satisfies the above conditions together with $\left\|\prod_{k=n}^{m} \phi_{k}(x)\right\| \geq(1-\epsilon)\|x\|$ for $x \in \mathscr{F}$. This implies that $\alpha$ is pseudo-diagonal.

\section{MF flows and dual MF flows}

Definition 3.1. Let $\left(k_{n}\right)$ be a sequence of positive integers and let $\beta_{n}$ be a flow on $M_{k_{n}}$. Let $\beta_{t}=\prod_{n=1}^{\infty} \beta_{n, t}$ which forms a (non-continuous) flow on $\prod_{n=1}^{\infty} M_{k_{n}}$. Let $\left(\prod_{n=1}^{\infty} M_{k_{n}}\right)_{\beta}$ denote the maximal $C^{*}$-subalgebra of $\prod_{n=1}^{\infty} M_{k_{n}}$ on which $\beta$ is continuous. A flow $\alpha$ on a separable $C^{*}$-algebra $A$ is called an $M F$ flow if there is an embedding of $A$ into $\left(\prod_{n=1}^{\infty} M_{k_{n}}\right)_{\beta} / \bigoplus_{n=1}^{\infty} M_{k_{n}}$ for some $\left(k_{n}\right)$ and $\left(\beta_{n}\right)$ such that $\beta_{t} \phi=\phi \alpha_{t}$.

We first state a technical lemma.

Lemma 3.2. There is a constant $C>0$ satisfying: Let $\alpha$ be a flow on a $C^{*}$-algebra A. If $e \in A$ is a projection such that $\max _{|t| \leq 1}\left\|\alpha_{t}(e)-e\right\|=\delta$ is sufficiently small, there is an $\alpha$-cocycle $u$ in $A$ (or in $A+\mathrm{C} 1$ if $A \not \ngtr 1)$ such that $\operatorname{Ad} u_{t} \alpha_{t}(e)=e$ and $\max _{|t| \leq 1}\left\|u_{t}-1\right\| \leq C \delta^{1 / 2}$.

Proof. Let $\delta_{\alpha}$ denote the generator of $\alpha$. If $e \in D\left(\delta_{\alpha}\right)$ then $e \delta_{\alpha}(e) e=$ $(1-e) \delta_{\alpha}(e)(1-e)=0$. Thus $[i h, e]=-\delta_{\alpha}(e)$ for $h=i\left(\delta_{\alpha}(e) e-e \delta_{\alpha}(e)\right)=$ $i(1-e) \delta_{\alpha}(e) e+i e \delta_{\alpha}(e)(1-e)$, which is a self-adjoint element of $A$ of norm less than or equal to $\left\|\delta_{\alpha}(e)\right\|$. Thus the differentiable $\alpha$-cocycle $u$ defined 
by $d u_{t} / d t=u_{t} \alpha_{t}(i h)$ satisfies the conditions that $\operatorname{Ad} u_{t} \alpha_{t}(e)=e$ and that $\max _{|t| \leq 1}\left\|u_{t}-1\right\| \leq\left\|\delta_{\alpha}(e)\right\|$.

If we only assume that $\max _{|t| \leq 1}\left\|\alpha_{t}(e)-e\right\|$ is small, we have to resort to the above situation. Namely we find a projection $e^{\prime} \in A$ such that $\left\|\delta_{\alpha}\left(e^{\prime}\right)\right\|$ is small and $e^{\prime}$ is close to $e$. Then finding a unitary $w \approx 1$ such that $w^{*} e w=e^{\prime}$ and an $\alpha$-cocycle $v$ such that $\operatorname{Ad} v_{t} \alpha_{t}\left(e^{\prime}\right)=e^{\prime}$ and $\left\|v_{t}-1\right\| \approx 0, t \in[-1,1]$, we would obtain the desired $\alpha$-cocycle $t \mapsto w v_{t} \alpha_{t}\left(w^{*}\right)$.

The following arguments are standard and mostly found in [4], but we shall give out some details (see the proof of Proposition 1.3 of [11]).

Let $e \in A$ be a projection and let $\delta=\max _{|t| \leq 1}\left\|\alpha_{t}(e)-1\right\|>0$.

Let $g$ be a non-negative $C^{\infty}$-function on R such that $g$ has compact support and $\int g(t) d t=1$. We define

$$
q=\int \delta^{1 / 2} g\left(\delta^{1 / 2} t\right) \alpha_{t}(e) d t,
$$

which satisfies that $0 \leq q \leq 1$. Since $\left\|\alpha_{t}(e)-e\right\| \leq \delta(1+|t|)$, we deduce that

$$
\|q-e\| \leq \int \delta^{1 / 2} g\left(\delta^{1 / 2} t\right)\left\|\alpha_{t}(e)-e\right\| d t \leq \delta+C_{1} \delta^{1 / 2} \leq\left(1+C_{1}\right) \delta^{1 / 2},
$$

where $C_{1}=\int g(t)|t| d t$. We assume that $\left(1+C_{1}\right) \delta^{1 / 2}<1 / 8$, which insures that $\operatorname{Sp}(q) \subset[0,1 / 8] \cup[7 / 8,1]$. Note that $q \in D\left(\delta_{\alpha}\right)$ and

$$
\left\|\delta_{\alpha}(q)\right\|=\left\|-\int \delta g^{\prime}\left(\delta^{1 / 2} t\right) \alpha_{t}(e) d t\right\| \leq C_{2} \delta^{1 / 2},
$$

where $C_{2}=\int\left|g^{\prime}(t)\right| d t$.

Let $f$ be a non-negative $C^{\infty}$-function on R such that $\operatorname{supp}(f) \subset[1 / 2,3 / 2]$ and $f(t)=1$ on $[7 / 8,1]$. Define $\hat{f}$ by $\hat{f}(p)=(2 \pi)^{-1} \int e^{-i p t} f(t) d t$ and set $C_{3}=\int|t \hat{f}(t)| d t$. We define

$$
e^{\prime}=f(q)=\int \hat{f}(t) e^{i t q} d t
$$

which is a projection such that $\left\|e^{\prime}-q\right\| \leq\left(1+C_{1}\right) \delta^{1 / 2}$. By Theorem 3.2.32 of [4] it follows that $e^{\prime} \in D\left(\delta_{\alpha}\right)$ and

$$
\left\|\delta_{\alpha}\left(e^{\prime}\right)\right\| \leq C_{3}\left\|\delta_{\alpha}(q)\right\| \leq C_{2} C_{3} \delta^{1 / 2} .
$$

Hence there is an $\alpha$-cocycle $v$ such that $\operatorname{Ad} v_{t} \alpha_{t}\left(e^{\prime}\right)=e^{\prime}$ and $\max _{|t| \leq 1} \| v_{t}-$ $1\|\leq\| \delta_{\alpha}\left(e^{\prime}\right) \| \leq C_{2} C_{3} \delta^{1 / 2}$.

Note that $\left\|e-e^{\prime}\right\| \leq\|e-q\|+\left\|q-e^{\prime}\right\| \leq 2\left(1+C_{1}\right) \delta^{1 / 2} \leq 1 / 4$. Since $\left\|e e^{\prime}+(1-e)\left(1-e^{\prime}\right)-1\right\| \leq 2\left\|e-e^{\prime}\right\| \leq 1 / 2$, the unitary $w$ obtained by the 
polar decomposition of $e e^{\prime}+(1-e)\left(1-e^{\prime}\right)$ satisfies that $\|w-1\| \leq 4\left\|e-e^{\prime}\right\|$. Since $w e^{\prime} w^{*}=e$, we conclude that the $\alpha$-cocycle $u: t \mapsto w v_{t} \alpha_{t}(w)^{*}$ satisfies that $\operatorname{Ad} u_{t} \alpha_{t}(e)=e$. Note that if $|t| \leq 1$, then $\left\|u_{t}-1\right\| \leq 2\|w-1\|+\left\|v_{t}-1\right\| \leq$ $\left(16+16 C_{1}+C_{2} C_{3}\right) \delta^{1 / 2}$. Thus if $\delta<8^{-2}\left(1+C_{1}\right)^{-2}$ then we obtain the desired cocycle $u$ for the constant $C=16+16 C_{1}+C_{2} C_{3}$.

Lemma 3.3. Let $\alpha$ be an MF flow on a unital separable $C^{*}$-algebra $A$. Then there is a unital embedding $\phi$ of $A$ into $\left(\prod M_{k_{n}}\right)_{\beta} / \bigoplus M_{k_{n}}$ such that $\phi \alpha_{t}=\beta_{t} \phi$ with $\beta=\prod \beta_{n}$ for some sequence $\left(k_{n}\right)$ in $\mathrm{N}$ and $\left(\beta_{n}\right)$.

Proof. Suppose that $A$ is embedded into $\left(\prod_{n=1}^{\infty} M_{k_{n}}\right)_{\beta} / \bigoplus_{n=1}^{\infty} M_{k_{n}}$ as in the definition. Let $\left(p_{n}\right) \in \prod M_{k_{n}}$ be a representative of the unit of $A$. We may suppose that $p_{n}^{*}=p_{n}$. Since $\left\|p_{n}^{2}-p_{n}\right\| \rightarrow 0$ we may also suppose that each $p_{n}$ is a projection by functional calculus. Since $\left\|\beta_{n, t}\left(p_{n}\right)-p_{n}\right\|$ converges to zero uniformly in $t \in[-1,1]$, there is a sequence $\left(u_{n, t}\right)$ of cocycles by Lemma 3.2 such that $u_{n, t}$ is a $\beta_{n}$-cocycle in $M_{k_{n}}$, Ad $u_{n, t} \beta_{n, t}\left(p_{n}\right)=p_{n}$, and $\left\|u_{n, t}-1\right\| \rightarrow 0$ uniformly in $t \in[-1,1]$. Thus we can replace $M_{k_{n}}$ by $p_{n} M_{k_{n}} p_{n}$ and $\beta_{n}$ by $\operatorname{Ad} u_{n, t} \beta_{n, t} \mid p_{n} M_{k_{n}} p_{n}$ and obtain the desired unital embedding.

Let $\mathscr{K}=\mathscr{K}\left(L^{2}(\mathrm{R})\right)$, the compact operators on $L^{2}(\mathrm{R})$, and define a unitary flow $\lambda$ on $L^{2}(\mathrm{R})$ by $\left(\lambda_{t} \xi\right)(s)=\xi(s-t), \xi \in L^{2}(\mathrm{R})$. We denote by $\operatorname{Ad} \lambda$ the flow on $\mathscr{K}$ defined by $t \mapsto \operatorname{Ad} \lambda_{t}$. The following proposition shows that there is a universal flow (on a non-separable $C^{*}$-algebra) for MF flows in the sense that the flow is MF if and only if it is realized as a subflow of the universal one.

The following is a technical lemma about almost commuting pairs of selfadjoint operators, one compact and the other possibly unbounded (cf. [12]).

Lemma 3.4. For every $\epsilon>0$ there is a $v>0$ satisfying the following condition: Let a $\in(\mathscr{K}(\mathscr{H}))_{s a}$ and $H$ a self-adjoint operator (which may be unbounded) on $\mathscr{H}$ such that $\|a\| \leq 1$ and $\|[a, H]\|<v$. Then there is an $a_{1} \in(\mathscr{K}(\mathscr{H}))_{\text {sa }}$ and a self-adjoint operator $H_{1}$ on $\mathscr{H}$ such that $a_{1}$ is of finite rank, $\left\|a-a_{1}\right\|<\epsilon,\left\|H-H_{1}\right\|<\epsilon, H-H_{1} \in \mathscr{K}(\mathscr{H})$, and $\left[a_{1}, H_{1}\right]=0$.

Proof. This follows from Theorem 3.1 of [2], where this is stated as a result valid for $a$ and $H$ on an arbitrary finite-dimensional space $\mathscr{H}$ without depending on the dimensionality.

Proposition 3.5. Let $\alpha$ be a flow on a separable $C^{*}$-algebra. Then the following conditions are equivalent.

(1) $\alpha$ is an MF flow.

(2) $(A, \alpha)$ can be embedded into $\left(\left(\prod \mathscr{K}_{n}\right)_{\gamma} / \bigoplus \mathscr{K}_{n}, \gamma\right)$, where $\mathscr{K}_{n}=\mathscr{K}$ and $\gamma=\prod \operatorname{Ad} \lambda$. 
Proof. Suppose (1), i.e., suppose that $(A, \alpha)$ can be embedded into

$$
\left(\prod M_{k_{n}}\right)_{\beta} / \bigoplus M_{k_{n}}
$$

with $\beta=\prod \beta_{n}$ for some $\left(k_{n}\right)$ and $\left(\beta_{n}\right)$. Let $v_{n}$ be a unitary flow in $M_{k_{n}}$ such that $\beta_{n, t}=\operatorname{Ad} v_{n, t}$. Then, since the spectrum of $\lambda$ is R, by using the Weylvon Neumann theorem one can obtain a sequence of $\lambda$-cocycles $u_{n}$ in $\mathscr{K}+\mathrm{C} 1$ and a sequence of finite-rank projections $e_{n} \in \mathscr{K}$ such that $u_{n, t}-1$ is compact, $\left\|u_{n, t}-1\right\| \rightarrow 0$ uniformly in $t \in[-1,1]$ as $n \rightarrow \infty, \operatorname{Ad}\left(u_{n, t} \lambda_{t}\right)\left(e_{n}\right)=e_{n}$, and the spectrum of $t \mapsto u_{n, t} \lambda_{t} e_{n}$ is equal to that of $v_{n}$ with multiplicity included. Then there is an embedding of $M_{k_{n}}$ into $e_{n} \mathscr{K}_{n} e_{n} \subset \mathscr{K}_{n}$ such that $v_{n}$ is mapped to $u_{n} \lambda e_{n}$. Thus one can embed $\left(\left(\prod M_{k_{n}}\right)_{\beta}, \beta\right)$ into $\left(\left(\prod \mathscr{K}_{n}\right)_{\sigma}, \sigma\right)$ with $\sigma=\prod\left(u_{n} \lambda\right)$. Since $u_{n, t} \rightarrow 1$ uniformly in $t$ on any bounded set of $\mathrm{R}$ and $u_{n, t}-1 \in \mathscr{K}_{n}$, one derives that $\prod u_{n, t} \in \bigoplus \mathscr{K}_{n}+\mathrm{C} 1$; thus $\sigma$ and $\gamma=\prod \lambda$ are equal on the quotient $\prod \mathscr{K}_{n} / \bigoplus \mathscr{K}_{n}$. Thus $(A, \alpha)$ can be embedded into $\left(\left(\prod \mathscr{K}_{n}\right)_{\gamma} / \bigoplus \mathscr{K}_{n}, \gamma\right)$.

Suppose (2). If $A$ is unital, this follows from the proof of Lemma 3.3. Suppose that $A$ is not unital. Let $\left(p_{k}\right)$ be an approximate identity for $A$ and let $\left(p_{k, n}\right)_{n} \in\left(\prod \mathscr{K}_{n}\right)_{\gamma}$ be a sequence representing $p_{k}$ with $0 \leq p_{k, n} \leq 1$. Let $f$ be a smooth non-negative function on R such that $\int f(t) d t=1$ and $\int\left|f^{\prime}(t)\right| d t$ is small. Note that $\left(\int f(t) \operatorname{Ad} \lambda_{t}\left(p_{k, n}\right) d t\right)_{n}$ represents $\alpha_{f}\left(p_{k}\right)=$ $\int f(t) \alpha_{t}\left(p_{k}\right) d t$ and that $\left\|\delta_{\alpha}\left(\alpha_{f}\left(p_{k}\right)\right)\right\| \leq \int\left|f^{\prime}(t)\right| d t$ etc., where $\delta_{\alpha}$ is the generator of $\alpha$. By using these facts we obtain a sequence $\left(e_{k}\right)$ in $A$ with $0 \leq e_{k} \leq 1$ and $\left(e_{k, n}\right)_{n} \in\left(\prod \mathscr{K}_{n}\right)_{\gamma}$ representing $e_{k}$ with $0 \leq e_{k, n} \leq 1$ such that $\left\|e_{k} x-x\right\| \rightarrow 0$ for any $x \in A,\left\|\delta_{\alpha}\left(e_{k}\right)\right\| \rightarrow 0$ as $k \rightarrow \infty$ and $\left\|\left[H, e_{k, n}\right]\right\| \rightarrow 0$ uniformly in $n$ as $k \rightarrow \infty$, where $H$ is the self-adjoint generator of $\lambda$.

Let $\left(x_{k}\right)$ be a dense sequence in the unit ball of $A_{s a}$ and let $\left(x_{k, n}\right)_{n}$ be a sequence of self-adjoint elements in the unit ball of $\left(\prod \mathscr{K}_{n}\right)_{\gamma}$ representing $x_{k}$. Let $n \in \mathrm{N}$. We choose $v>0$ for $\epsilon=2^{-n}$ as in Lemma 3.4. We choose $k \in \mathrm{N}$ such that $\left\|e_{k} x_{i}-x_{i}\right\|<\epsilon$ for any $i \leq n$ and $\left\|\left[H, e_{k, m}\right]\right\|<v$ for all $m \in \mathbf{N}$. We choose $M_{n} \in \mathrm{N}$ such that $\left\|e_{k, m} x_{i, m}-x_{i, m}\right\|<\epsilon$ for all $m \geq M_{n}$. Then by Lemma 3.4 we choose a self-adjoint $H_{m}$ on $L^{2}(\mathrm{R})$ and a finite-rank selfadjoint operator $e_{k, m}^{\prime}$ for $m \geq M_{n}$ such that $\left[H_{m}, e_{k, m}^{\prime}\right]=0, H_{m}-H$ is compact, $\left\|H_{m}-H\right\|<\epsilon$, and $\left\|e_{k, m}-e_{k, m}^{\prime}\right\|<\epsilon$. Let $P_{m}$ be the support projection of $e_{k, m}^{\prime}$. Then $P_{m}$ is a finite-rank projection commuting with $H_{m}$ and satisfies that $\left\|P_{m} x_{i, m}-x_{i, m}\right\| \leq 2 \epsilon+\left\|P_{m} e_{k, m}^{\prime} x_{i, m}-x_{i, m}\right\|=2 \epsilon+\left\|e_{k, m}^{\prime} x_{i, m}-x_{i, m}\right\| \leq$ $4 \epsilon=2^{-n+2}$ for $i \leq n$. We may suppose that $\left(M_{n}\right)$ is strictly increasing and we make such a choice for $M_{n} \leq m<M_{n+1}$ and set $B_{m}=P_{m} \mathscr{K} P_{m}$ and $\beta_{m, t}=$ Ad $e^{i t H_{m}} \mid B_{m}$. Then it follows that $\left(P_{m} x_{i, m} P_{m}\right)$ is equal to $\left(x_{i, m}\right)$ modulo $\bigoplus \mathscr{K}_{m}$ for all $i$ and $\gamma_{t}^{\prime}=\prod \operatorname{Ad} e^{i t H_{m}}=\operatorname{Ad} u_{t} \gamma_{t}$ for some $\gamma$-cocycle $u$ with $u_{t}-1 \in$ 
$\bigoplus \mathscr{K}_{m}$. Hence $(A, \alpha)$ can be embedded into $\left(\prod B_{m}\right)_{\beta} / \bigoplus B_{m}$ equipped with $\beta=\prod \beta_{m}$ which is embedded into $\left(\prod \mathscr{K}_{m}\right)_{\gamma^{\prime}} / \bigoplus \mathscr{K}_{m}=\left(\prod \mathscr{K}_{m}\right)_{\gamma} / \bigoplus \mathscr{K}_{m}$ equipped with $\gamma$ such that the composition is the original embedding of $(A, \alpha)$. This completes the proof.

REMARK 3.6. In the above proposition the property we needed for $\lambda$ is that its spectrum contains arbitrarily long intervals of $R$.

Proposition 3.7. The class of MF flows on a separable $C^{*}$-algebra is closed under cocycle perturbations.

Proof. Let $\alpha$ be an MF flow on $A$ and let $u$ be an $\alpha$-cocycle. Let $\phi$ be an embedding of $A$ into $\left(\prod M_{k_{n}}\right)_{\beta} / \bigoplus M_{k_{n}}$ such that $\phi \alpha_{t}=\beta_{t} \phi$ for some $\left(k_{n}\right)$ and $\beta=\prod \beta_{n}$.

If $A$ is unital, then $u_{t}$ belongs to $A$ and we may assume that $\phi$ is unital. By Lemma 1.1 of [7] it follows that $u$ is given as $w u_{t}^{(h)} \alpha_{t}\left(w^{*}\right)$, where $w$ is a unitary and $u^{(h)}$ is the differentiable $\alpha$-cocycle defined by $d u_{t}^{(h)} /\left.d t\right|_{t=0}=i h$ with $h=h^{*} \in A$. Then we find a $\beta$-cocycle $v$ in $\left(\prod M_{k_{n}}\right)_{\beta}$, by lifting $w$ and $h$ to a unitary and a self-adjoint element respectively, such that $v_{t}=\prod v_{n, t}$ maps to $\phi\left(u_{t}\right)$ under the quotient map. Hence we obtain that $\phi \operatorname{Ad} u_{t} \alpha_{t}=\beta_{t}^{\prime} \phi$ with $\beta_{t}^{\prime}=\prod \operatorname{Ad} v_{n, t} \beta_{n, t}$ (regarded as a flow on the quotient).

If $A$ is not unital and $u$ is an $\alpha$-cocycle in the multiplier algebra $M(A)$ of $A$, we approximate $u$ by $\alpha$-cocycles in $A+\mathrm{C} 1$ [9]. More precisely let $\left(x_{i}\right)$ be a dense sequence in $A$ and let $\left(u^{(n)}\right)$ be a sequence of $\alpha$-cocycles in $A+\mathrm{C} 1$ such that

$$
\left\|\left(u_{t}-u_{t}^{(n)}\right) x_{i}\right\| \leq 2^{-n}\left\|x_{i}\right\|, \quad t \in[-1,1]
$$

for $i=1,2, \ldots, n$. We extend $\phi$ to a $\mathrm{CP}$ map from $A+\mathrm{C} 1$ into $\left(\prod M_{k_{n}}\right)_{\beta} / \bigoplus M_{k_{n}}$ by setting $\phi(1)=1$. We then lift each $\phi\left(u^{(n)}\right)$ to an $\beta$ cocycle $v^{(n)}$ in $\left(\prod M_{k_{n}}\right)_{\beta}$ as stated above. We also fix a lifting $y_{i} \in\left(\prod M_{k_{n}}\right)_{\beta}$ of each $\phi\left(x_{i}\right)$. We then have for $i \leq n$

$$
\left\|Q\left(\left(v_{t}^{(n)}-v_{t}^{(n+1)}\right) y_{i}\right)\right\| \leq\left(2^{-n}+2^{-n-1}\right)\left\|x_{i}\right\|, \quad t \in[-1,1],
$$

where $Q$ is the quotient map onto $\left(\prod M_{k_{n}}\right)_{\beta} / \bigoplus M_{k_{n}}$. Hence one can choose a sequence $\left(K_{n}\right)$ of integers such that $K_{0}=0, K_{n}>K_{n-1}$, and

$$
\sup _{k \geq K_{n}}\left\|\left(v_{k, t}^{(n)}-v_{k, t}^{(n+1)}\right) y_{i, k}\right\| \leq 2^{-n+1}\left\|x_{i}\right\|, \quad t \in[-1,1]
$$

for $i \leq n$. We define a $\beta$-cocycle $w \in\left(\prod M_{k_{n}}\right)_{\beta}$ by $w_{k, t}=v_{k, t}^{(n)}$ for $K_{n} \leq k<$ $K_{n+1}$. If $m>n$ and $K_{m} \leq k<K_{m+1}$ then the norm of the $k^{\prime}$ th coordinate of $\left(w_{t}-v_{t}^{(n)}\right) y_{i}$ is

$$
\left\|v_{k, t}^{(m)} y_{i, k}-v_{k, t}^{(n)} y_{i, k}\right\| \leq 2^{-n+2}\left\|x_{i}\right\|
$$


for $i \leq n$. Hence it follows that $\left\|Q\left(w_{t}\right) \phi\left(x_{i}\right)-\phi\left(u_{t}^{(n)} x_{i}\right)\right\|=\| Q\left(w_{t} y_{i}-\right.$ $\left.v_{t}^{(n)} y_{i}\right)\left\|\leq 2^{-n+2}\right\| x_{i} \|$ for $i \leq n$, which implies that $\left\|Q\left(w_{t}\right) \phi\left(x_{i}\right)-\phi\left(u_{t} x_{i}\right)\right\| \leq$ $2^{-n+3}\left\|x_{i}\right\|$. Since $n$ is arbitrary, we can conclude that $Q\left(w_{t}\right) \phi(a)=\phi\left(u_{t} a\right)$ for any $a \in A$. We replace the flow $\beta_{n}$ on $M_{k_{n}}$ by $t \mapsto \operatorname{Ad} w_{n, t} \beta_{n, t}$. Then it follows that $\phi \operatorname{Ad} u_{t} \alpha_{t}=\beta_{t} \phi$.

A $*$-linear generalized inductive system of flows is a sequence of flows $\left(A_{n}, \alpha_{n}\right)$ together with $*$-linear maps $\phi_{m, n}: A_{m} \rightarrow A_{n}$ for $m<n$ with $\phi_{m, n} \phi_{k, m}=\phi_{k, n}$ for all $k<m<n$ such that for all $k$ and all $x, y \in A_{k}$ and $\epsilon>0$ there is an $K>k$ such that for all $n>m \geq K$ and $t \in[-1,1]$

(1) $\left\|\phi_{m, n}\left(\phi_{k, m}(x) \phi_{k, m}(y)\right)-\phi_{k, n}(x) \phi_{k, n}(y)\right\|<\epsilon$,

(2) $\left\|\phi_{m, n} \alpha_{m, t} \phi_{k, m}(x)-\alpha_{n, t} \phi_{k, n}(x)\right\|<\epsilon$,

(3) $\sup _{r>m}\left\|\phi_{m r} \mid L\left(\left\{\alpha_{m, t} \phi_{k m}(x)|| t \mid<\delta\right\}\right)\right\|<\infty$,

for some $\delta>0$, where $L(S)$ is the linear span of $S$.

This notion and the following consequences are adapted from Section 2 of [1]. The above condition 3 replaces $\sup _{r>k}\left\|\phi_{k, r}(x)\right\|<\infty$ there.

For such a system one defines the inductive limit $C^{*}$-algebra $A$ and the flow $\alpha$ on $A$, which may be realized as follows. Let $\prod_{n=1}^{\infty} A_{n}$ be the full $C^{*}$-direct product of the $A_{n}$ 's and let $\beta_{t}=\prod_{n=1}^{\infty} \alpha_{n, t}$. Let $\bigoplus_{n=1}^{\infty} A_{n}$ be the $C^{*}$-direct sum, the ideal of $\prod_{n=1}^{\infty} A_{n}$ consisting of sequences converging to zero in norm. Define a map $\phi_{m}$ of $A_{m}$ into $\prod A_{n}$ by $\phi_{m}(x)_{n}=\phi_{m, n}(x)$ for $n \geq m$ and 0 for $n<m$. Since $\phi_{m}(x)-\phi_{n} \phi_{m, n}(x) \in \bigoplus A_{n}$ one can define a $*$-linear map $\phi$ of $\bigcup A_{n}$ into $\prod A_{n} / \bigoplus A_{n}$ by $\phi \mid A_{m}=Q \phi_{m}$, where $Q$ is the quotient map of $\prod M_{k_{n}}$ onto $\prod M_{k_{n}} / \bigoplus M_{k_{n}}$. Since $\phi(x) \phi(y)=Q\left(\phi_{m}(x) \phi_{m}(y)\right)$ is the limit of $\phi\left(\phi_{m, n}(x) \phi_{m, n}(y)\right)$ as $n \rightarrow \infty$ for $x, y \in A_{m}, \phi$ extends to an isomorphism of the inductive limit $A$ of the system $\left(A_{n}, \phi_{m n}\right)$ into $\prod A_{n} / \bigoplus A_{n}$. Now we could identify the inductive limit $A$ with the closure of $\phi\left(\bigcup A_{n}\right)$.

Since $Q \beta_{t} \phi_{m}(x)=Q\left(\left(\alpha_{n, t} \phi_{m, n}(x)\right)_{n}\right)$ is the limit of $\phi \alpha_{n, t} \phi_{m, n}(x)$ as $n \rightarrow$ $\infty$ for $x \in A_{m}, \beta_{t}$ induces an automorphism of $A$ which we denote by $\alpha_{t}$.

We shall show that $t \mapsto \alpha_{t} \phi(x)$ is continuous for $x \in A_{m}$. Let $\epsilon>0$. Then there is $M>m$ such that for $n>\ell \geq M$ and $t \in[-1,1]$ we have that $\left\|\phi_{\ell, n} \alpha_{\ell, t} \phi_{m, \ell}(x)-\alpha_{n, t} \phi_{m, n}(x)\right\|<\epsilon$. Hence $\left\|\alpha_{t} \phi(x)-\phi(x)\right\| \leq$ $\left\|\phi\left(\alpha_{\ell, t} \phi_{m, \ell}(x)-\phi_{m, \ell}(x)\right)\right\|+\epsilon$. Fixing $\ell \geq M$ there is a $1>\delta>0$ such that if $|t|<\delta$ then $\left\|\alpha_{\ell, t} \phi_{m, \ell}(x)-\phi_{m, \ell}(x)\right\|<\epsilon$. Hence we obtain that if $|t|<\delta$ then $\left\|\alpha_{t} \phi(x)-\phi(x)\right\|<2 \epsilon$. Thus $\alpha$ is a (continuous) flow. Note that $\alpha$ is realized as the restriction of $\beta=\prod \alpha_{n}$.

Let $\left(\prod A_{n}\right)_{\beta}$ be the maximal $C^{*}$-subalgebra of $\prod A_{n}$ on which $\beta$ is continuous. We note that the image of $\phi_{m}$ is contained in $\left(\prod A_{n}\right)_{\beta}$. Suppose that there are a sequence $\left(t_{i}\right)$ in $\mathrm{R}$, a sequence $\left(n_{i}\right)$ in $\mathrm{N}, x \in A_{m}$, and a $\delta>0$ 
such that $\lim _{i} t_{i}=0$ and $\left\|\alpha_{n_{i}, t_{i}} \phi_{m, n_{i}}(x)-\phi_{m, n_{i}}(x)\right\|>\delta$. Since each $\alpha_{n}$ is continuous we must have that $n_{i} \rightarrow \infty$. Note that there is $\ell>m$ such that $\left\|\phi_{\ell, n} \alpha_{\ell, t} \phi_{m, \ell}(x)-\alpha_{n, t} \phi_{m, n}(x)\right\|<\delta / 2$ for $t \in[-1,1]$ and

$$
\sup _{r>\ell} \| \phi_{\ell, r} \mid L\left(\left\{\alpha_{\ell, t} \phi_{m, \ell}(x)|| t \mid<s\right\} \|<\infty\right.
$$

for some $s>0$. Since $\left\|\phi_{\ell, n_{i}}\left(\alpha_{\ell, t_{i}} \phi_{m, \ell}(x)-\phi_{m, \ell}(x)\right)\right\|>\delta / 2$ for $n_{i}>\ell$, this contradicts that $t \mapsto \alpha_{\ell, t}$ is continuous. Hence one concludes that $\phi$ embeds $A$ into $\left(\prod A_{n}\right)_{\beta} / \bigoplus A_{n}$.

Lemma 3.8. Suppose that $(A, \alpha)$ can be embedded into $\left(\left(\prod M_{k_{n}}\right)_{\beta} / \bigoplus M_{k_{n}}\right.$, $\beta)$ with $\beta=\prod \beta_{n}$. Then there exist a (separable) $C^{*}$-algebra $B$ on a separable Hilbert space $\mathscr{H}$ and a unitary flow $U$ on $\mathscr{H}$ such that $B$ includes $\mathscr{K}(\mathscr{H})$, $t \mapsto \operatorname{Ad} U_{t}(x)$ defines a flow on $B$, there is an isomorphism $\phi$ of $B / \mathscr{K}(\mathscr{H})$ onto A such that $\phi Q \operatorname{Ad} U_{t}(x)=\alpha_{t} \phi Q(x)$ for $x \in B$, and $(B, U)$ is quasidiagonal, where $Q$ is the quotient map of $B$ onto $B / \mathscr{K}(\mathscr{H})$. Conversely if there is such $(B, U)$ then $(A, \alpha)$ can be embedded into $\left(\left(\prod M_{k_{n}}\right)_{\beta} / \bigoplus M_{k_{n}}, \beta\right)$ for some $\left(k_{n}\right)$ and $\left(\beta_{n}\right)$.

Proof. Let $\mathscr{H}$ be an infinite-dimensional separable Hilbert space and let $\left(E_{n}\right)$ be a sequence of projections on $\mathscr{H}$ such that $E_{n} \mathscr{H}$ is $k_{n}$-dimensional, $E_{m} E_{n}=0$ for $m \neq n$, and $\sum_{n=1}^{\infty} E_{n}=1$. Let $\sigma$ be a map of $A$ into $\left(\prod M_{k_{n}}\right)_{\beta}$ such that $Q^{\prime} \sigma$ is the given embedding of $A$ into $\left(\prod M_{k_{n}}\right)_{\beta} / \bigoplus M_{k_{n}}$, where $Q^{\prime}$ is the quotient map of $\prod M_{k_{n}}$ onto $\prod M_{k_{n}} / \bigoplus M_{k_{n}}$. We identify $E_{n} \mathscr{B}(\mathscr{H}) E_{n}$ with $M_{k_{n}}$ and denote by $\iota$ the embedding of $\prod M_{k_{n}}$ into $\mathscr{B}(\mathscr{H})$ by $\iota(x)=\sum_{n=1}^{\infty} x_{n}$ for $x=\left(x_{n}\right)_{n}$. Note that $\iota$ induces the embedding of $\prod M_{k_{n}} / \bigoplus M_{k_{n}}$ into $\mathscr{B}(\mathscr{H}) / \mathscr{K}(\mathscr{H})$ since $\iota\left(\prod M_{k_{n}}\right) \cap \mathscr{K}(\mathscr{H})=\iota\left(\bigoplus M_{k_{n}}\right)$. We let $\psi=\iota \sigma$, which is a map of $A$ into $\mathscr{B}(\mathscr{H})$.

Let $B=\psi(A)+\mathscr{K}(\mathscr{H})$, which is a quasi-diagonal $C^{*}$-algebra such that $Q \psi$ is an isomorphism of $A$ onto $B / \mathscr{K}(\mathscr{H})$. Thus $\phi$ is obtained as the inverse of $Q \psi$.

Let $U_{n}$ be a unitary flow in $M_{k_{n}}=E_{n} \mathscr{B}(\mathscr{H}) E_{n}$ such that $\operatorname{Ad} U_{n, t}=\beta_{n, t}$ and let $U_{t}=\iota\left(\left(U_{n, t}\right)_{n}\right)$ which is a unitary flow in $\mathscr{B}(\mathscr{H})$ such that $t \mapsto U_{t}$ is strongly continuous. Note that $t \mapsto \operatorname{Ad} U_{t}(x)$ is norm-continuous for $x \in B$. Then we have for $x \in A$ that $Q \operatorname{Ad} U_{t} \psi(x)=Q \iota \beta_{t} \sigma(x)=Q \psi \alpha_{t}(x)$, where we use $Q^{\prime} \beta_{t} \sigma=Q^{\prime} \sigma \alpha_{t}$ and $Q \iota=0$ on $\operatorname{Ker} Q^{\prime}$. Since $\phi=(Q \psi)^{-1}$, we obtain that $\phi Q \operatorname{Ad} U_{t} \psi(x)=\alpha_{t}(x)$. For $y=\psi(x)+c$ with $c \in \mathscr{K}(\mathscr{H})$ we obtain that $\phi Q \operatorname{Ad} U_{t}(y)=\phi \operatorname{Ad} U_{t} \psi(x)=\alpha_{t}(x)=\alpha_{t} \phi Q(y)$. This concludes the proof of the first part.

Conversely if there is such a $(B, U)$ then there is an increasing sequence $\left(P_{n}\right)$ of finite-rank projections on $\mathscr{H}$ such that $\lim _{n} P_{n}=1,\left\|\left[P_{n}, U_{t}\right]\right\| \rightarrow 0$ 
uniformly in $t \in[-1,1]$, and $\left\|\left[P_{n}, b\right]\right\| \rightarrow 0$ for $b \in B$. We may suppose that $\left[P_{n}, U_{t}\right]=0$ by perturbing of $U$ by compacts and passing to a subsequence of $\left(P_{n}\right)$. Set $E_{n}=P_{n}-P_{n-1}$ and $k_{n}=$ rank $E_{n}$ with $P_{0}=0$. Identifying $E_{n} \mathscr{B}(\mathscr{H}) E_{n}$ with $M_{k_{n}}$ we define a map $\phi: B \rightarrow \prod M_{k_{n}}$ by $\phi(x)=\left(E_{n} x E_{n}\right)_{n}$. This drops to a $*$-homomorphism of $B$ into $\left(\prod M_{k_{n}}\right)_{\beta} / \bigoplus M_{k_{n}}$, intertwining $\alpha$ with $\beta$, whose kernel is exactly $\mathscr{K}(\mathscr{H})$.

A continuous field of flows over $\mathrm{N} \cup\{\infty\}$ is a continuous field of $C^{*}$ algebras $A_{n}, n \in \mathrm{N} \cup\{\infty\}$ and flows $\alpha_{n}$ on $A_{n}$ such that if $n \mapsto x_{n}$ is a continuous field so is $n \mapsto \alpha_{n, t}\left(x_{n}\right)$ for all $t \in \mathrm{R}$. Since $\left\|x_{n}-\alpha_{n, t}\left(x_{n}\right)\right\|$ converges to $\left\|x_{\infty}-\alpha_{\infty, t}\left(x_{\infty}\right)\right\|$ as $n \rightarrow \infty$ in $\mathrm{N}$, it follows that $t \mapsto \alpha_{n, t}\left(x_{n}\right)$ is continuous uniformly in $n \in \mathrm{N} \cup\{\infty\}$. Hence if $n \mapsto x_{n}$ is a continuous field then so is $n \mapsto \int f(t) \alpha_{n, t}\left(x_{n}\right) d t$ for $f \in L^{1}(\mathrm{R})$. Note also that the flow $\alpha=\prod_{n=1}^{\infty} \alpha_{n} \times \alpha_{\infty}$ defined on the $C^{*}$-algebra generated by the continuous fields is strongly continuous.

We will present a version of Proposition 2.2.3 of [1] by borrowing the terminology there; a finite product $\prod_{n=r}^{s}\left(A_{n}, \alpha_{n}\right)$ for $1 \leq r \leq s<\infty$ is called a segment of $\prod_{n=1}^{\infty}\left(A_{n}, \alpha_{n}\right)$ and two segments are disjoint if their intersection is zero when they are naturally regarded as subsystems of $\prod_{n=1}^{\infty}\left(A_{n}, \alpha_{n}\right)$.

Lemma 3.9. Let $\alpha_{n}$ be a flow on a separable $C^{*}$-algebra $A_{n}$ and $\beta=$ $\prod_{n=1}^{\infty} \alpha_{n}$. Let $(A, \alpha)$ be a flow with $A$ separable. Then the following are equivalent:

(1) $(A, \alpha)$ can be embedded into $\left(\left(\prod A_{n}\right)_{\beta} / \bigoplus A_{n}, \beta\right)$.

(2) There is a continuous field of flows $\left(B_{n}, \beta_{n}\right)$ over $\mathrm{N} \cup\{\infty\}$ such that $\left(B_{n}, \beta_{n}\right)$ is a segment of $\prod\left(A_{n}, \alpha_{n}\right)$ for $n \in \mathrm{N}$ with disjoint segments for different $n$ and such that $\left(B_{\infty}, \beta_{\infty}\right) \cong(A, \alpha)$.

(3) $(A, \alpha)$ can be embedded into $\left(\left(\prod B_{n}\right)_{\gamma} / \oplus B_{n}, \gamma\right)$, where $\left(B_{n}, \beta_{n}\right)$ is a segment of $\prod\left(A_{n}, \alpha_{n}\right)$ for $n \in \mathrm{N}$ with disjoint segments for different $n$ and $\gamma=\prod \beta_{n}$, such that $\|x\|=\lim _{n}\left\|x_{n}\right\|$ holds for every $x \in A$ and sequence $\left(x_{n}\right)$ representing $x$.

Proof. We follow the proof of Proposition 2.2.3 of [1].

We shall prove $(1) \Rightarrow(2)$ as follows: Let $\left(x_{i}\right)$ be a dense sequence in $A$ with $\left(x_{i n}\right)_{n} \in\left(\prod A_{n}\right)_{\beta}$ representing $x_{i}$ and let $\left(t_{j}\right)$ be an enumeration of the rationals. For $i, j$ and $n \in \mathbf{N} \cup\{\infty\}$ we set $y_{i, j}(n)=\alpha_{n, t_{i}}\left(x_{j, n}\right) \in A_{n}$ for $n \in \mathbf{N}$ and $y_{i, j}(\infty)=\alpha_{t_{i}}\left(x_{j}\right) \in A_{\infty}=A$. Let $P$ be the set of all polynomials in noncommuting variables $Y_{i, j}, i, j \in \mathrm{N}$ and their formal adjoints $Y_{i, j}^{*}, i, j \in \mathrm{N}$ with coefficients in $\mathrm{Q}+i \mathrm{Q}$. Since $P$ is countable, let $\left(f_{i}\right)$ be a fixed enumeration of $P$. For $n \in \mathbf{N} \cup\{\infty\}$ we set $f_{i}(n)$ to be the element in $A_{n}$ obtained from $f_{i}$ substituting $Y_{i, j}=y_{i, j}(n)$ for all $i, j$. 
There are disjoint segments $\left[r_{m}, s_{m}\right]$ in $\mathrm{N}$ such that for $i=1,2, \ldots, m$

$$
\left\|f_{i}(\infty)\right\|-\left\|\prod_{n=r_{m}}^{s_{m}} f_{i}(n)\right\| \mid<1 / m .
$$

Set $B(m)=\prod_{n=r_{m}}^{s_{m}} A_{n}$ and $\beta_{m, t}=\prod_{n=r_{m}}^{s_{m}} \alpha_{n, t}$. We set $F_{i}(m)=\prod_{n=r_{m}}^{s_{m}} f_{i}(n) \in$ $B(m)$ and $F_{i}(\infty)=f_{i}(\infty)$. Then the function $n \mapsto\left\|F_{i}(n)\right\|$ is continuous on $\mathrm{N} \cup\{\infty\}$ and the set of $F_{i}$ 's, together with the sequences converging to zero, forms a $*$-algebra $\mathscr{A}$ over $\mathrm{Q}+i \mathrm{Q}$ invariant under $\prod_{m=1}^{\infty} \beta_{m, t} \times \alpha_{t}, t \in \mathrm{Q}$. Since $\left(x_{i n}\right)_{n} \in\left(\prod A_{n}\right)_{\beta}, m \mapsto \beta_{m, t_{j}} F_{i}(m)-F_{i}(m) \in \mathscr{A}$ converges to zero uniformly in $m \in \mathrm{N}$ as $t_{j} \rightarrow 0$. Hence the closure of $\mathscr{A}$ is a $C^{*}$-algebra invariant under $\prod \beta_{m} \times \alpha$ on which $t \mapsto \prod \beta_{m, t} \times \alpha_{t}$ is continuous. Thus the continuous fields are invariant under the flow.

For the other implications see the proof of Proposition 2.2.3 in [1].

The following result will be proved by mimicking the proof of Theorem 3.2.2 of [1].

Theorem 3.10. Let $\alpha$ be a flow on a separable $C^{*}$-algebra A. Then the following conditions are equivalent:

(1) $(A, \alpha)$ is obtained as the inductive limit of a $*$-linear generalized inductive system of flows on finite-dimensional $C^{*}$-algebras.

(2) $\alpha$ is an MF flow.

(3) There is an essential quasi-diagonal extension $B$ of $A$ by the compact operators $\mathscr{K}$ and a unitary flow $U \in M(\mathscr{K})$ such that $\operatorname{Ad} U_{t}(B)=B$ for $t \in \mathrm{R}, t \mapsto \operatorname{Ad} U_{t}(x)$ is norm-continuous for $x \in B,(B, U)$ is quasi-diagonal and $Q \operatorname{Ad} U_{t}=\alpha_{t} Q$ where $Q$ is the quotient map of $B$ onto A.

(4) There is a continuous field of flows $\left(A_{n}, \alpha_{n}\right)$ over $\mathrm{N} \cup\{\infty\}$ such that $A_{n}$ is finite-dimensional for $n \in \mathrm{N}$ and $\left(A_{\infty}, \alpha_{\infty}\right) \cong(A, \alpha)$.

(5) There is a continuous field of flows $\left(A_{n}, \alpha_{n}\right)$ over $\mathrm{N} \cup\{\infty\}$ such that $A_{n} \cong M_{k_{n}}$ for some $k_{n}$ for each $n \in \mathrm{N}$ and $\left(A_{\infty}, \alpha_{\infty}\right) \cong(A, \alpha)$.

Proof. We proved (1) $\Rightarrow$ (2) before Lemma 3.8 and (2) $\Leftrightarrow$ (3) in Lemma 3.8 and (2) $\Leftrightarrow(4)$ in Lemma 3.9. (5) $\Rightarrow(4)$ is trivial and (4) $\Rightarrow(5)$ is easy since the fibres at any isolated points may be enlarged.

It remains to show (2) $\Rightarrow(1)$. Suppose that $(A, \alpha)$ is embedded into

$$
\left(\prod M_{k_{n}}\right)_{\beta} / \bigoplus M_{k_{n}}
$$

with $\beta=\prod \beta_{n}$ for some $\left(M_{k_{n}}, \beta_{n}\right)$. For $x \in A$ let $\mathrm{Sp}_{\alpha}(x)$ denote the $\alpha$ spectrum of $x$ and let $A^{\alpha}(F)=\left\{x \in A \mid \operatorname{Sp}_{\alpha}(x) \subset F\right\}$ for a closed set 
$F$ of R. Let $A_{C}=\bigcup_{n=1}^{\infty} A^{\alpha}[-n, n]$, which is a dense $*$-subalgebra of $A$. Similarly let $\left(\prod M_{k_{n}}\right)_{C}=\left(\left(\prod M_{k_{n}}\right)_{\beta}\right)_{C}=\bigcup_{n=1}^{\infty}\left(\prod M_{k_{n}}\right)^{\beta}[-n, n]$, where $\left(\prod M_{k_{n}}\right)^{\beta}[-n, n]=\left(\left(\prod M_{k_{n}}\right)_{\beta}\right)^{\beta}[-n, n]$. For each $x \in A_{C}$ there is a $\left(x_{n}\right) \in$ $\left(\prod M_{k_{n}}\right)_{\beta}$ representing $x$. If $f \in L^{1}(\mathrm{R})$ has Fourier transform with compact support and is 1 on the $\alpha$-spectrum of $x$ then we have that $\int f(t) \alpha_{t}(x) d t=x$, which implies that $\int f(t) \beta_{t}\left(\left(x_{n}\right)\right) d t$ also represents $x$. In this way we deduce that $A^{\alpha}[-n, n]$ is embedded into $\left(\prod M_{k_{n}}\right)^{\beta}[-n-1, n+1] / \bigoplus M_{k_{n}}$. Note that $A^{\alpha}[-n, n]$ etc. are self-adjoint. We choose a $*$-linear map $\sigma$ of $A_{C}$ into $\left(\prod M_{k_{n}}\right)_{C}$ such that $Q \sigma=\mathrm{id}$ on $A_{C}$ and $\sigma\left(A^{\alpha}[-n, n]\right) \subset\left(\prod M_{k_{n}}\right)^{\beta}[-n-$ $1, n+1]$. We also choose a dense sequence $\left(x_{n}\right)$ in $A_{C}$.

We shall define finite-dimensional $C^{*}$-algebras $A_{n}$ with flows $\alpha_{n}$ on $A_{n}$ and *-linear maps $\gamma_{n}: A_{n} \rightarrow A_{C} \subset A$ and $\delta_{n}: A \rightarrow A_{n+1}$ such that the sequence $\left(A_{n}, \alpha_{n}\right)$ of flows with maps $\phi_{n, n+1} \equiv \phi_{n}=\delta_{n} \gamma_{n}: A_{n} \rightarrow A_{n+1}$ is a $*$-linear generalized inductive system of flows with the desired properties, appearing as the upper sequence of the commutative diagram:

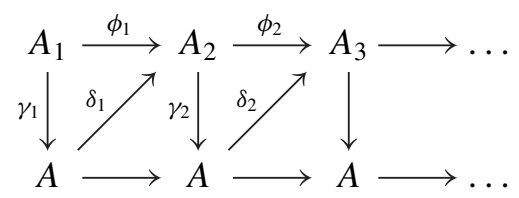

In particular our system will satisfy the following conditions:

$$
\left\|\phi_{n+1}(x y)-\phi_{n+1}(x) \phi_{n+1}(y)\right\| \leq 2^{-n}\|x\|\|y\|
$$

for all $x, y \in \phi_{n}\left(A_{n}\right) \subset A_{n+1}$ and

$$
\left\|\phi_{n+1} \alpha_{n+1, t}(x)-\alpha_{n+2, t} \phi_{n+1}(x)\right\| \leq 2^{-n}\|x\|
$$

for all $x \in \phi_{n}\left(A_{n}\right)$ and $t \in[-1,1]$, which is enough to imply that the system has the desired properties together with the condition $\sup _{n>k}\left\|\phi_{k, n}\right\|<\infty$ for $k \in \mathrm{N}$.

On the other hand the lower sequence of copies of $(A, \alpha)$ of the above commutative diagram with maps $\gamma_{n+1} \delta_{n}: A \rightarrow A$ defines $(A, \alpha)$, which follows from: $\left(\gamma_{n}\left(A_{n}\right)\right)$ is increasing with dense union in $A, \gamma_{n+1} \delta_{n}(x)=x$ for $x \in \gamma_{n}\left(A_{n}\right)$, and

$$
\left\|\gamma_{n+1} \delta_{n} \alpha_{t}(x)-\alpha_{t} \gamma_{n+1} \delta_{n}(x)\right\| \leq 2^{-n+1}\|x\|
$$

for $x \in \gamma_{n}\left(A_{n}\right)$ and $t \in[-1,1]$. We shall require the intertwining properties for $\delta_{n}$ and $\gamma_{n}$ with $\alpha_{n}$ and $\alpha$, which will imply that both the upper and lower sequences define the same object, i.e., $(A, \alpha)$. 
In the course of the inductive construction below we shall define a finitedimensional *-subspace $V_{n}$ of $A_{C} \subset A$ depending on $\gamma_{n}$ which is a vital ingredient for constructing $A_{n+1}, \delta_{n}$ and then $\gamma_{n+1}$ such that $\left(V_{n}\right)$ forms an increasing sequence with dense union in $A$. In particular the norms of $\delta_{n}$ and $\gamma_{n+1}$ will be almost dominated by $\left(\operatorname{dim} V_{n}\right)^{1 / 2}$ and $V_{n}$ will equal $\gamma_{n+1}\left(A_{n+1}\right)$. To obtain the above inequalities we shall require the following properties for $\gamma_{n}$ and $\delta_{n}$ with $n \geq 2$. The first two are discussed in the proof of Theorem 3.2.2 of [1] and the second two are new being concerned with the flows:

$$
\left\|\delta_{n}(x y)-\delta_{n}(x) \delta_{n}(y)\right\| \leq 2^{-n-1}\left(\operatorname{dim} V_{n}\right)^{-1 / 2}\|x\|\|y\|
$$

for all $x, y \in \gamma_{n}\left(A_{n}\right)$,

$$
\gamma_{n+1} \delta_{n}(x)=x
$$

for all $x \in \gamma_{n}\left(A_{n}\right) \cdot \gamma_{n}\left(A_{n}\right)$ or $x \in \gamma_{n}\left(A_{n}\right)$,

$$
\left\|\alpha_{n+1, t} \delta_{n}(x)-\delta_{n} \alpha_{t}(x)\right\| \leq 2^{-n}\|x\|
$$

for $x \in \gamma_{n}\left(A_{n}\right)$, and

$$
\left\|\alpha_{t} \gamma_{n+1}(x)-\gamma_{n+1} \alpha_{n+1, t}(x)\right\| \leq 2^{-n-1}\|x\|
$$

for $x \in \phi_{n}\left(A_{n}\right)$.

We set $A_{1}=\mathrm{C}$ and $\gamma_{1}: A_{1} \rightarrow A_{C}$ be an arbitrary $*$-linear map. Suppose that

$$
A_{2}, \delta_{1}, \gamma_{2}, A_{3}, \delta_{2}, \gamma_{3}, \ldots, A_{n}, \delta_{n-1}, \gamma_{n}
$$

are constructed so that $\gamma_{k}\left(A_{k-1}\right) \subset \gamma_{k}\left(A_{k}\right)$ and $x_{k-1} \in \gamma_{k}\left(A_{k}\right)$ for $k \leq n$ as well as the above inequalities, where $\left(x_{n}\right)$ was chosen as a dense sequence in $A_{C}$. We shall define $A_{n+1}$, and $\delta_{n}: A \rightarrow A_{n+1}$, and $\gamma_{n+1}: A_{n+1} \rightarrow A_{C}$.

Let $d$ be the dimension of $A_{n}$. Let $E \in \mathrm{N}$ be such that $\operatorname{Sp}_{\alpha}(x) \subset[-E, E]$ and $\operatorname{Sp}_{\beta} \sigma(x) \subset[-E, E]$ for all $x \in \gamma_{n}\left(A_{n}\right)$, which exists by the assumption on $\gamma_{n}$ and $\sigma$. We choose $N \in \mathrm{N}$ such that $E \sqrt{d(2 N+1)+d^{2}+2} / N<$ $2^{-n-3}$. Let $V_{n}$ be the $*$-subspace of $A$ generated by $\gamma_{n}\left(A_{n}\right) \cdot \gamma_{n}\left(A_{n}\right), x_{n}, x_{n}^{*}$ and $\alpha_{k / N}\left(\gamma_{n}\left(A_{n}\right)\right)$ with $k=0, \pm 1, \pm 2, \ldots, \pm N$. Note that $V_{n} \subset A_{C}$ and the $\operatorname{dim}\left(V_{n}\right) \leq d(2 N+1)+d^{2}+2$. Note also that $\alpha_{t}(x)$ with $x \in \gamma_{n}\left(A_{n}\right), t \in$ $[-1,1]$ is almost contained in $V_{n}$; more precisely, there is a $y \in V_{n}$ such that $\left\|\alpha_{t}(x)-y\right\| \leq(E / N)\|x\|$. This follows by setting $y=\alpha_{k / N}(x)$ for some $k$ due to the estimate: $\left\|\alpha_{s}(x)-\alpha_{t}(x)\right\| \leq E|s-t|\|x\|$, which is derived from $\operatorname{Sp}_{\alpha}(x) \subset[-E, E]$.

Since $Q \sigma=$ id and $Q \beta_{t} \sigma=\alpha_{t}$ we will then choose $r_{n}<s_{n}$ such that the linear map $\rho_{n}: A \rightarrow A_{n+1} \equiv \prod_{i=r_{n}}^{s_{n}} M_{k_{i}}$ defined by $x \mapsto \prod_{i=r_{n}}^{s_{n}} \sigma_{i}(x)$ satisfies the following conditions: $\rho_{n} \mid V_{n}$ is almost isometric and $\rho_{n} \mid \gamma_{n}\left(A_{n}\right)$ is 
almost multiplicative and $\alpha_{n+1, t} \rho_{n} \equiv \prod_{i=r_{n}}^{s_{n}} \beta_{i, t} \rho_{n}$ is nearly equal to $\rho_{n} \alpha_{t}$ on $\gamma_{n+1}\left(V_{n}\right)$, i.e., for any prescribed $\epsilon>0$,

$$
\begin{aligned}
\left\|\rho_{n} \mid V_{n}\right\| & <1+\epsilon, \\
\left\|\left(\rho_{n} \mid V_{n}\right)^{-1}\right\| & <1+\epsilon, \\
\left\|\rho_{n}(x) \rho_{n}(y)-\rho_{n}(x y)\right\| & \leq \epsilon\|x\|\|y\|, \quad x, y \in \gamma_{n}\left(A_{n}\right), \\
\left\|\alpha_{n+1, k / N} \rho_{n}(x)-\rho_{n} \alpha_{k / N}(x)\right\| & \leq \epsilon\|x\|, \quad x \in \gamma_{n}\left(A_{n}\right), \quad k=0, \pm 1, \ldots, \pm N .
\end{aligned}
$$

Let $P_{n}$ be a projection from $A$ onto $V_{n}$ such that $\left\|P_{n}\right\| \leq \sqrt{\operatorname{dim} V_{n}}$ (see 1.14 of [13]; we need this stronger estimate rather than $\left\|P_{n}\right\| \leq \operatorname{dim} V_{n}$ ). We set $\delta_{n}=$ $\rho_{n} P_{n}: A \rightarrow A_{n+1}$. Let $R_{n}$ be a projection from $A_{n+1}$ onto $\delta_{n}(A)=\rho_{n}\left(V_{n}\right)$ such that $\left\|R_{n}\right\| \leq \sqrt{\operatorname{dim} V_{n}}$ and set $\gamma_{n+1}=\left(\rho_{n} \mid V_{n}\right)^{-1} R_{n}: A_{n+1} \rightarrow A_{C}$. Then it is immediate that $\gamma_{n+1} \delta_{n} \mid V_{n}=\mathrm{id}$. We set

$$
\epsilon=2^{-n-3}\left(\operatorname{dim} V_{n}\right)^{-1 / 2},
$$

which assures the first inequalities on $\delta_{n}$.

Note that $\gamma_{n+1}\left(A_{n+1}\right)=\left(\rho_{n} \mid V_{n}\right)^{-1}\left(\rho_{n}\left(V_{n}\right)\right)=V_{n}$, which implies that $\gamma_{n+1}\left(A_{n+1}\right) \supset \gamma_{n}\left(A_{n}\right)$ and $\gamma_{n+1}\left(A_{n+1}\right) \ni x_{n}$.

We have defined $\phi_{n}=\delta_{n} \gamma_{n}: A_{n} \rightarrow A_{n+1}$. Since

$$
\gamma_{n} \delta_{n-1}=\left(\rho_{n-1} \mid V_{n-1}\right)^{-1} R_{n-1} \rho_{n-1} P_{n-1}=P_{n-1}
$$

is a projection onto $V_{n-1}$ and the range of $\gamma_{m}$ is $V_{m-1}$, we obtain that $\phi_{m, n}=$ $\phi_{n-1} \phi_{n-2} \ldots \phi_{m}=\delta_{n-1} \gamma_{m}=\rho_{n-1}\left(\rho_{m-1} \mid V_{m-1}\right)^{-1} R_{m-1}$, i.e., $\left\|\phi_{m, n}\right\|<$ $4 \sqrt{\operatorname{dim} V_{m-1}}$ for all $n>m$.

Let us repeat here the proof from [1] for $\phi_{n+1}$ being approximately multiplicative. For $x, y \in A_{n}$, since $\phi_{n, n+2}=\delta_{n+1} \gamma_{n}, \| \phi_{n+1}\left(\phi_{n}(x) \phi_{n}(y)\right)-$ $\phi_{n, n+2}(x) \phi_{n, n+2}(y) \|$ is less than or equal to

$$
\begin{aligned}
& \left\|\delta_{n+1}\left\{\gamma_{n+1}\left(\delta_{n} \gamma_{n}(x) \delta_{n} \gamma_{n}(y)\right)-\gamma_{n}(x) \gamma_{n}(y)\right\}\right\| \\
& +\left\|\delta_{n+1}\left(\gamma_{n}(x) \gamma_{n}(y)\right)-\delta_{n+1} \gamma_{n}(x) \delta_{n+1} \gamma_{n}(y)\right\| .
\end{aligned}
$$

Substituting $\gamma_{n}(x) \gamma_{n}(y)=\gamma_{n+1} \delta_{n}\left(\gamma_{n}(x) \gamma_{n}(y)\right)$ the first term is less than or equal to

$$
\left\|\delta_{n+1} \gamma_{n+1}\right\|\left\|\delta_{n} \gamma_{n}(x) \delta_{n} \gamma_{n}(y)-\delta_{n}\left(\gamma_{n}(x) \gamma_{n}(y)\right)\right\|
$$

which is roughly smaller than $2^{-n-1}\left\|\gamma_{n}(x)\right\|\left\|\gamma_{n}(y)\right\|$. The second term is roughly smaller than $2^{-n-2}\left(\operatorname{dim} V_{n}\right)^{-1 / 2}\left\|\gamma_{n}(x)\right\|\left\|\gamma_{n}(y)\right\|$. Thus one can estimate that

$$
\left\|\phi_{n+1}\left(\phi_{n}(x) \phi_{n}(y)\right)-\phi_{n, n+2}(x) \phi_{n, n+2}(y)\right\| \leq 2^{-n}\left\|\phi_{n}(x)\right\|\left\|\phi_{n}(y)\right\| .
$$


Now we come to the proof of the intertwining properties of $\delta_{n}$ and $\gamma_{n+1}$ with $\alpha_{t}, \alpha_{n+1, t}$.

Let $x \in \gamma_{n}\left(A_{n}\right)$. For $t \in[-1,1]$ we want to estimate $\left\|\delta_{n} \alpha_{t}(x)-\alpha_{n+1, t} \delta_{n}(x)\right\|$. First assume that $t=k / N$ with $k \in[-N, N]$. Since $\alpha_{t}(x), x \in V_{n}$ we have $\left\|\delta_{n} \alpha_{t}(x)-\alpha_{n+1, t} \delta_{n}(x)\right\|=\left\|\rho_{n} \alpha_{t}(x)-\alpha_{n+1, t} \rho_{n}(x)\right\| \leq \epsilon\|x\|$. If $t \in[-1,1]$ in general there is $k / N$ such that $|t-k / N|<1 / N$. Since

$$
\begin{aligned}
\left\|\delta_{n} \alpha_{t}(x)-\delta_{n} \alpha_{k / N}(x)\right\| & \leq\left\|\delta_{n}\right\|\left\|\alpha_{t}(x)-\alpha_{k / N}(x)\right\| \\
& \leq(1+\epsilon) \sqrt{\operatorname{dim} V_{n}} E N^{-1}\|x\|
\end{aligned}
$$

and

$$
\left\|\alpha_{n+1, t} \delta_{n}(x)-\alpha_{n+1, k / N} \delta_{n}(x)\right\| \leq E / N\left\|\rho_{n}(x)\right\| \leq(1+\epsilon) E N^{-1}\|x\|
$$

and $\sqrt{\operatorname{dim} V_{n}} E N^{-1}<2^{-n-3}$, we obtain that

$$
\left\|\delta_{n} \alpha_{t}(x)-\alpha_{n+1, t} \delta_{n}(x)\right\| \leq\left(\epsilon+2^{-n-2}(1+\epsilon)\right)\|x\| \leq 2^{-n}\|x\| .
$$

Let $x \in V_{n}$ and $t=k / N$. Since $\gamma_{n+1} \rho_{n}(x)=x$ and $\gamma_{n+1} \rho_{n} \alpha_{t}(x)=\alpha_{t}(x)$, we have

$$
\left\|\alpha_{t} \gamma_{n+1} \rho_{n}(x)-\gamma_{n+1} \alpha_{n+1, t} \rho_{n}(x)\right\|=\left\|\gamma_{n+1}\left(\rho_{n} \alpha_{t}(x)-\alpha_{n+1, t} \rho_{n}(x)\right)\right\|
$$

which is less than or equal to $\epsilon\left\|\gamma_{n+1}\right\|\|x\| \leq(1+\epsilon)^{2} \epsilon \sqrt{\operatorname{dim} V_{n}}\left\|\rho_{n}(x)\right\|$. If $t \in[-1,1]$ in general there is $k$ such that $|t-k / N|<1 / N$. Since

$$
\left\|\alpha_{t} \gamma_{n+1} \rho_{n}(x)-\alpha_{k / N} \gamma_{n+1} \rho_{n}(x)\right\|=\left\|\alpha_{t}(x)-\alpha_{k / N}(x)\right\|
$$

is less than or equal to $(1+\epsilon) E N^{-1}\left\|\rho_{n}(x)\right\| \leq(1+\epsilon) 2^{-n-3}\left\|\rho_{n}(x)\right\|$ and

$$
\left\|\gamma_{n+1} \alpha_{n+1, t} \rho_{n}(x)-\gamma_{n+1} \alpha_{n+1, k / N} \rho_{n}(x)\right\|
$$

is less than or equal to $\left\|\gamma_{n+1}\right\| E N^{-1}\left\|\rho_{n}(x)\right\| \leq(1+\epsilon) \sqrt{\operatorname{dim} V_{n}} E N^{-1}\left\|\rho_{n}(x)\right\|$, we obtain that

$$
\begin{aligned}
& \left\|\alpha_{t} \gamma_{n+1} \rho_{n}(x)-\gamma_{n+1} \alpha_{n+1, t} \rho_{n}(x)\right\| \\
& \quad \leq\left(2(1+\epsilon) 2^{-n-3}+(1+\epsilon)^{2} \epsilon \sqrt{\operatorname{dim} V_{n}}\right)\left\|\rho_{n}(x)\right\|,
\end{aligned}
$$

which is less than or equal to $2^{-n-1}\left\|\rho_{n}(x)\right\|$. This completes the proof.

REMARK 3.11. In the above proof (2) $\Rightarrow$ (1) of the theorem we have chosen a lifting $\sigma$ of $A \subset\left(\prod M_{k_{n}}\right)_{\beta} / \bigoplus M_{k_{n}}$ such that $\sigma\left(A_{C}\right) \subset\left(\prod M_{k_{n}}\right)_{C}$ and constructed $V_{n}$ in $A_{C}$. (We actually defined $\sigma$ only on $A_{C}$.) We could have chosen a $\sigma$ such that $\sigma\left(D\left(\delta_{\alpha}\right)\right) \subset D\left(\delta_{\beta}\right)$, where $\delta_{\beta}$ is the generator of $\beta$ 
(on $\left.\left(\prod M_{k_{n}}\right)_{\beta}\right)$, and constructed $V_{n}$ in $D\left(\delta_{\alpha}\right)$. Because what we needed was Lipschitz continuity of $t \mapsto \alpha_{t}(x)$ and $t \mapsto \beta_{t} \sigma(x)$ of $x \in \gamma_{n}\left(A_{n}\right)=V_{n-1}$.

Corollary 3.12. Let $\alpha$ be an MF flow on a separable $C^{*}$-algebra $A$. Then there is an $R F$ flow $(B, \beta)$ and a $\beta$-invariant ideal I such that the quotient of $(B, \beta)$ by $I$ is isomorphic to $(A, \alpha)$.

Proof. Let $B$ be the $C^{*}$-algebra generated by the continuous fields as in Condition (5) of Theorem 3.10 applied to $(A, \alpha)$, which has the flow $\beta$ determined by $\alpha_{n}, n \in \mathrm{N} \cup\{\infty\}$. Let $\pi_{n}$ be the representation of $B$ which picks up the fiber $M_{k_{n}}$ at $n \in \mathrm{N}$. Then the family $\pi_{n}, n \in \mathrm{N}$ is faithful and each $\pi_{n}$ is $\beta$-covariant, i.e., $\beta$ is an RF flow. Let $I$ be the ideal of $B$ generated by the fields $n \mapsto a_{n}$ with $a_{\infty}=0$. (Note that $I=\bigoplus_{n=1}^{\infty} M_{k_{n}}$ is $\beta$-invaraint.) Then the quotient of $(B, \beta)$ by $I$ is isomorphic to $(A, \alpha)$.

The following is about KMS states.

Proposition 3.13. Let $B=\prod_{n=1}^{\infty} M_{k_{n}}$ and $I=\bigoplus M_{k_{n}}$ for some $\left(k_{n}\right)$ and $\beta_{t}=\prod \beta_{n, t}$. The flow $\beta$ on $B_{\beta} / I$ has KMS states for all inverse temperatures.

Proof. Fix an inverse temperature. Then each $\beta_{n}$ has a unique KMS state $\omega_{n}$ on $M_{k_{n}}$. Let $\mathcal{U}$ be an ultra filter on $\mathrm{N}$ and define a state $\omega$ on $B_{\beta}$ by $\omega\left(\left(x_{n}\right)\right)=$ $\lim _{n \rightarrow \mathscr{U}} \omega_{n}\left(x_{n}\right)$, which is a KMS state and satisfies that $\omega \mid I=0$. Thus we may regard $\omega$ as a state of $B_{\beta} / I$.

Corollary 3.14. Let $\alpha$ be an MF flow on a unital separable $C^{*}$-algebra. Then $\alpha$ has KMS states for all inverse temperatures.

Proof. There is a unital embedding of $(A, \alpha)$ into $\left(\prod M_{k_{n}}\right)_{\beta} / \bigoplus M_{k_{n}}$ by 3.3. Hence this follows from the previous proposition.

From now on we are concerned with the dual object of MF flows.

LEMMA 3.15. If there is a continuous field of flows $\left(B_{n}, \beta_{n}\right)$ over $\mathrm{N} \cup\{\infty\}$ then there is a continuous field of flows $\left(B_{n} \times{ }_{\beta_{n}} \mathrm{R}, \hat{\beta}_{n}\right)$ over $\mathrm{N} \cup\{\infty\}$ such that if $n \mapsto x_{n}$ is a continuous field for the former and $f \in L^{1}(\mathrm{R})$ then $n \mapsto x_{n} \lambda_{n}(f)$ is a continuous field for the latter, where $\lambda_{n}$ is the natural embedding of $L^{1}(\mathrm{R})$ into $M\left(B_{n} \times_{\beta_{n}} \mathrm{R}\right)$.

Proof. Let $x_{i} \in B_{\infty}$ and $f_{i} \in L^{1}(\mathrm{R})$ for $i=1,2, \ldots, k$. Let $\left(x_{i, n}\right)$ be a continuous field with $x_{i, \infty}=x_{i}$. We shall show that $\left\|\sum_{i=1}^{k} x_{i, n} \lambda_{n}\left(f_{i}\right)\right\|$ converges to $\left\|\sum_{i=1}^{k} x_{i} \lambda\left(f_{i}\right)\right\|$ as $n \rightarrow \infty$, where $\lambda=\lambda_{\infty}$. Since $\hat{\beta}_{n, p}\left(x_{i n} \lambda_{n}\left(f_{i}\right)\right)=$ $x_{i n} \lambda_{n}\left(\chi_{p} f_{i}\right)$ with $\chi_{p}(t)=e^{i p t}$, this suffices to conclude the proof.

Let $\rho\left(\sum_{i=1}^{k} x_{i} \lambda\left(f_{i}\right)\right)=\lim \sup _{n}\left\|\sum_{i=1}^{k} x_{i n} \lambda_{n}\left(f_{i}\right)\right\|$. Since

$$
\rho\left(\sum_{i=1}^{k} x_{i} \lambda\left(f_{i}\right)\right) \leq \int\left\|\sum x_{i} f_{i}(t)\right\| d t,
$$


$\rho$ is well-defined on the $L^{1}$-closure of the linear span of $x \lambda(f), x \in B_{\infty}$, $f \in L^{1}(\mathrm{R})$. Since $\left(\sum x_{i n} \lambda_{n}\left(f_{i}\right)\right)^{*}\left(\sum x_{i n} \lambda_{n}\left(f_{i}\right)\right)=\sum_{i, j} \int \beta_{n,-t}\left(x_{j n}^{*} x_{i n}\right) \bar{f}_{j}(t)$ $\lambda_{n}(t)^{*} d t \lambda\left(f_{i}\right)$ can be approximated in $L^{1}$ norm by $\sum_{i, j} \sum_{\ell} \beta_{n,-t_{\ell}}\left(x_{j n}^{*} x_{i n}\right)$ $\lambda\left(f_{j} \Delta_{\ell}\right)^{*} \lambda\left(f_{i}\right)$ uniformly in $n$, where $\Delta_{\ell}$ 's are non-negative functions supported around $-t_{\ell}$ such that $\left(\sum_{\ell} \Delta_{\ell}\right) f_{j} \approx f_{j}$ in $L^{1}$ norm for all $j$, one can conclude that

$$
\rho\left(\left(\sum x_{i} \lambda(f)\right)^{*}\left(\sum x_{i} \lambda\left(f_{i}\right)\right)\right)=\rho\left(\sum x_{i} \lambda\left(f_{i}\right)\right)^{2} .
$$

Hence $\rho$ is a $C^{*}$-semi-norm. Since $\rho \hat{\beta}_{\infty, p}=\rho$, if $\rho$ is not a norm it vanishes on the ideal generated by a non-zero ideal of $B_{\infty}$. If $x$ is a non-zero element of that ideal and $\left(x_{n}\right)$ is a continuous field with $x_{\infty}=x$, then it should follow that $\lim _{n}\left\|x_{n} \lambda_{n}(f)\right\|=0$ for any $f \in L^{1}(\mathrm{R})$. Since $t \mapsto \beta_{n, t}\left(x_{n}\right)$ is continuous uniformly in $n$ we may suppose that the $\beta_{n}$-spectrum of $x_{n}$ is contained in $(-1,1)$ for all $n$. If $\hat{f}$ is 1 on $[0,1]$ one deduces $\left\|x_{n} \lambda(f)\right\| \geq\left\|x_{n}\right\| / 3$, which contradicts that $x \neq 0$. (Assuming $B_{n} \times_{\beta_{n}} \mathrm{R}$ is faithfully represented, let $E$ be the spectral measure of $t \mapsto \lambda_{t}$ and set $P_{i}=E(i-1, i]$. Since $x_{n}=\sum_{i} P_{i+1} x P_{i}+\sum_{i} P_{i} x_{n} P_{i}+\sum_{i} P_{i-1} x_{n} P_{i}$ one deduces that one of the three terms has at least norm $\left\|x_{n}\right\| / 3$. Note that the norm of the first term is $\sup \left\|P_{i+1} x_{n} P_{i}\right\|=\left\|P_{1} x_{n} P_{0}\right\| \leq\left\|x_{n} P_{0}\right\|$ using the fact that the norm is invariant under the dual flow. With similar formulas for other terms one reaches the conclusion.) Thus one can conclude that $\rho$ is the $C^{*}$-norm on $B_{\infty} \times_{\beta_{\infty}} \mathrm{R}$. Since the same arguments apply to any subsequence one concludes that $\lim _{n}\left\|\sum x_{i n} \lambda\left(f_{i}\right)\right\|=\left\|\sum x_{i} \lambda\left(f_{i}\right)\right\|$.

Definition 3.16. Let $\left(k_{n}\right)$ be a sequence of positive integers and let $\gamma_{n}$ be the flow on $M_{k_{n}} \otimes C_{0}(\mathrm{R})$ induced from translations, i.e., $\left(\gamma_{n, t} f\right)(s)=f(s-t)$ for $f \in M_{k_{n}} \otimes C_{0}(\mathrm{R})=C_{0}\left(\mathrm{R}, M_{k_{n}}\right)$. A flow $\alpha$ on a separable $C^{*}$-algebra is called a dual MF flow if there is such a sequence $\left(k_{n}\right)$ and an embedding of $(A, \alpha)$ into $\left(\prod_{n=1}^{\infty} M_{k_{n}} \otimes C_{0}(\mathrm{R})\right)_{\gamma} / \bigoplus M_{k_{n}} \otimes C_{0}(\mathrm{R})$ equipped with $\gamma=\prod \gamma_{n}$.

Proposition 3.17. The class of dual MF flows on a separable $C^{*}$-algebra is closed under cocycle perturbations.

Proof. This is proved in the same way as Proposition 3.7 once we notice the following: Any $\gamma$-cocycle $u$ in $M\left(M_{k} \otimes C_{0}(\mathrm{R})\right)$ is a coboundary. In fact if we set $w(s)=u_{s}(s)$ for such a $\gamma$-cocycle $u$ then $w \in M\left(M_{k} \otimes C_{0}(\mathrm{R})\right)$ and $w \gamma_{t}\left(w^{*}\right)(s)=w(s) w(s-t)^{*}=u_{s}(s) u_{s-t}(s-t)^{*}=u_{t}(s)$.

We provide some details. Let $(A, \alpha)$ be a dual MF flow and $\phi$ an embedding of $(A, \alpha)$ into $\left(\prod_{n=1}^{\infty} M_{k_{n}} \otimes C_{0}(\mathrm{R})\right)_{\gamma} / \bigoplus M_{k_{n}} \otimes C_{0}(\mathrm{R})$. Note that $A$ is nonunital (see 3.21 below) and let $u$ be an $\alpha$-cocycle in $M(A)$. If $\left(x_{i}\right)$ is a dense 
sequence in $A$ there is a sequence $\left(u^{(n)}\right)$ of $\alpha$-cocycles in $A+\mathrm{Cl}$ [9] such that

$$
\left\|\left(u_{t}-u_{t}^{(n)}\right) x_{i}\right\| \leq 2^{-n}\left\|x_{i}\right\|, \quad t \in[-1,1], \quad i=1,2, \ldots, n .
$$

There are self-adjoint $h_{n}, b_{n} \in A+\mathrm{C} 1$ such that $u_{t}^{(n)}=e^{i b_{n}} u_{t}^{\left(h_{n}\right)} \alpha_{t}\left(e^{-i b_{n}}\right)$ (see Lemma 1.1 of [7]). By lifting $\tilde{\phi}\left(h_{n}\right), \tilde{\phi}\left(b_{n}\right)$ to self-adjoint elements in

$$
\left(\prod_{n=1}^{\infty} M_{k_{n}}\right)_{\gamma} \otimes C_{0}(\mathrm{R})+\mathrm{C} 1,
$$

where $\tilde{\phi}$ is the unitization of $\phi$, we obtain a $\gamma$-cocycle $v^{(n)}$ in $\left(\prod_{n=1}^{\infty} M_{k_{n}}\right)_{\gamma} \otimes$ $C_{0}(\mathrm{R})+\mathrm{C} 1$ such that $Q\left(v_{t}^{(n)}\right)=u_{t}^{(n)}$, where $Q$ is the quotient map. We write $v_{t}^{(n)}=\left(v_{k, t}^{(n)}\right)$, where $v_{k}^{(n)}$ is a $\gamma_{n}$-cocycle in $M_{k_{n}} \otimes C_{0}(\mathrm{R})+\mathrm{C} 1$. By patching up these $v_{k}^{(n)}$ we can construct a $\gamma$-cocycle $w$ in $\prod_{n=1}^{\infty}\left(M_{k_{n}} \otimes C_{0}(\mathrm{R})+\mathrm{C} 1\right)$ such that $Q\left(w_{t}\right) \phi(a)=\phi\left(u_{t} a\right)$ for all $a \in A$ (see the proof of 3.6 for details). Then we conclude that $\left(\operatorname{Ad} w_{t} \gamma_{t}\right)^{-} \phi(a)=\phi\left(\operatorname{Ad} u_{t} \alpha_{t}(a)\right), a \in A$, where $\left(\operatorname{Ad} w_{t} \gamma_{t}\right)^{-}$is the flow on the quotient induced by $\operatorname{Ad} w_{t} \gamma_{t}$. Since $w_{t}$ is given as $U \gamma_{t}(U)^{*}$ with a unitary $U$ in $\prod_{n=1}^{\infty} M\left(M_{k_{n}} \otimes C_{0}(\mathrm{R})\right)$, it follows that $\left(\gamma_{t}\right)^{-} \operatorname{Ad} Q\left(U^{*}\right) \phi(a)=\operatorname{Ad} Q\left(U^{*}\right) \phi\left(\operatorname{Ad} u_{t} \alpha_{t}(a)\right), a \in A$. Thus the embedding $\operatorname{Ad} Q\left(U^{*}\right) \phi$ intertwines $\operatorname{Ad} u_{t} \alpha_{t}$ with $\gamma_{t}$ concluding the proof that $\operatorname{Ad} u \alpha$ is a dual MF flow.

Lemma 3.18. Let $\alpha$ be a flow on a separable $C^{*}$-algebra $A$.

(1) If $\alpha$ is an MF flow, then $\hat{\alpha}$ is a dual MF flow on $A \times{ }_{\alpha} \mathrm{R}$.

(2) If $\alpha$ is a dual MF flow, then $\hat{\alpha}$ is an MF flow on $A \times{ }_{\alpha} \mathrm{R}$.

Proof. By Theorem 3.10 if $\alpha$ is an MF flow then there is a continuous field of flows $\left(A_{n}, \alpha_{n}\right)$ over $\mathrm{N} \cup\{\infty\}$ such that $A_{n}=M_{k_{n}}$ for $n \in \mathbf{N}$ and $\left(A_{\infty}, \alpha_{\infty}\right) \cong(A, \alpha)$. Hence by Lemma 3.15 there is a continuous field of flows $\left(A_{n} \times_{\alpha_{n}} \mathrm{R}, \hat{\alpha}_{n}\right)$. Since $\left(A_{n} \times_{\alpha_{n}} \mathrm{R}, \hat{\alpha}_{n}\right) \cong\left(M_{k_{n}} \otimes C_{0}(\mathrm{R}), \gamma_{n}\right)$ one concludes that $\left(A \times{ }_{\alpha} \mathrm{R}, \hat{\alpha}\right)$ is a dual MF flow, where $\gamma_{n}$ is induced from translations.

If $\alpha$ is a dual MF flow then there is a continuous field of flows $\left(B_{n}, \gamma_{n}\right)$ over $\mathrm{N} \cup\{\infty\}$ such that $B_{n}=M_{k_{n}} \otimes C_{0}(\mathrm{R})$ and $\gamma_{n}$ is induced from translations for $n \in \mathrm{N}$ and $\left(B_{\infty}, \gamma_{\infty}\right) \cong(A, \alpha)$. Then by Lemma 3.15 we obtain a continuous field of flows $\left(B_{n} \times \gamma_{n} \mathrm{R}, \hat{\gamma}_{n}\right)$. Note that $B_{n} \times \gamma_{n} \mathrm{R} \cong M_{k_{n}} \otimes \mathscr{K}$ and $\hat{\gamma}_{n}=\mathrm{id} \otimes \operatorname{Ad} \lambda$ for $n \in \mathrm{N}$. Hence by Proposition 3.5 (and the remark after that) we conclude that $\left(A \times_{\alpha} \mathrm{R}, \hat{\alpha}\right)$ is MF.

Proposition 3.19. Let $\alpha$ be a flow on a separable $C^{*}$-algebra. Then $\alpha$ is an MF flow (resp. a dual MF flow) if and only if $\hat{\alpha}$ is a dual MF flow (resp. an MF flow). 
Proof. The "only if" part is shown in the above lemma. Suppose that $\hat{\alpha}$ is a dual MF flow. Then $\hat{\hat{\alpha}}$ is an MF flow by the above lemma, i.e., we conclude that $\alpha \otimes \operatorname{Ad} \lambda$ is an MF flow on $A \otimes \mathscr{K}$ by the Takesaki-Takai duality. Hence $\alpha \otimes$ id is also an MF flow on $A \otimes \mathscr{K}$ by 3.7; thus $\alpha$ is because $A \otimes e$ is an $\alpha \otimes$ id-invariant $C^{*}$-subalgebra of $A \otimes \mathscr{K}$, where $e$ is a minimal projection in $\mathscr{K}$.

Suppose that $\hat{\alpha}$ is an MF flow. Then $\hat{\hat{\alpha}}=\alpha \otimes \operatorname{Ad} \lambda$ is a dual MF flow on $A \otimes \mathscr{K}$. Then one concludes that $\alpha$ is a dual MF flow just as above.

Proposition 3.20. Let $\alpha$ be a flow on a separable $C^{*}$-algebra $A$. Then the following conditions are equivalent.

(1) $\alpha$ is a dual MF flow.

(2) $(A, \alpha)$ can be embedded into $\left(\left(\prod_{n=1}^{\infty} \mathscr{K}_{n} \otimes C_{0}(\mathrm{R})\right)_{\gamma} / \bigoplus \mathscr{K}_{n} \otimes C_{0}(\mathrm{R}), \gamma\right)$, where $\mathscr{K}_{n}=\mathscr{K}, \gamma=\prod \gamma_{n}$, and $\gamma_{n}$ is the flow induced by translations.

Proof. (1) $\Rightarrow(2)$ is easy. Suppose (2). Then one derives that $\left(A \times_{\alpha} R, \hat{\alpha}\right)$ satisfies the condition (2) in Proposition 3.5 since the crossed product of $C_{0}(\mathrm{R})$ by translations is $\mathscr{K}$. Hence $\hat{\alpha}$ is an MF flow. Thus $\alpha$ is a dual MF flow.

Remark 3.21. If $\alpha$ is a dual MF flow on $A$, then $A$ has no non-zero projections because $\mathscr{K} \otimes C_{0}(\mathrm{R})$ has no non-zero projections. In particular $A$ has no unit. If $\alpha$ is a dual MF flow then no $\alpha_{t} \neq$ id is approximately inner (i.e., no sequence of unitaries in $A+\mathrm{C} 1$ approximates $\alpha_{t}$ by adjoint action).

Here we give some examples. The flow $\gamma$ on $\mathscr{K} \otimes C_{0}(\mathrm{R})$ induced by translations is not a MF flow (see Example 2.10 in [11]) but of course it is a dual MF flow. The flow $\operatorname{Ad} \lambda$ on $\mathscr{K}$ is an MF flow but not a dual MF flow. (By the duality given in 3.19 these two statements are equivalent, giving another proof of Example 2.10 quoted above.) The identity flow on $\mathscr{K} \otimes C_{0}(\mathrm{R})$ is both an MF flow and a dual MF flow. (It is quasi-diagonal. To see that it is a dual MF flow define an isomorphism $\phi$ of $\mathscr{K} \otimes C_{0}(\mathrm{R})$ into $\prod_{n=1}^{\infty} \mathscr{K}_{n} \otimes C_{0}(\mathrm{R})$ by $\phi(f)=\left(f_{1}, f_{2}, \ldots\right)$ with $f_{n}(t)=f(t / n)$ for $f \in \mathscr{K}_{n} \otimes C_{0}(\mathrm{R}) \cong C_{0}(\mathrm{R}, \mathscr{K})$. Then $\phi$ embeds $\left(\mathscr{K} \otimes C_{0}(\mathrm{R})\right.$, id) into $\left(\prod \mathscr{K}_{n} \otimes C_{0}(\mathrm{R}) / \bigoplus \mathscr{K}_{n} \otimes C_{0}(\mathrm{R}), \gamma\right)$. From this it follows that id $\otimes \mathrm{id} \otimes \gamma$ on $\mathscr{K} \otimes C_{0}(\mathrm{R}) \otimes C_{0}(\mathrm{R})$ is both an MF flow and a dual MF flow.

\section{NF flows}

The condition in the following lemma is a flow version of (vi) of Theorem 5.2.2 of [1].

Lemma 4.1. Let $A$ be a nuclear $C^{*}$-algebra and $\alpha$ a quasi-diagonal flow on $A$. Then for any finite subset $\mathscr{F}$ of $A$ and $\epsilon>0$ there is a flow $\beta$ on a finitedimensional $C^{*}$-algebra $B$ and completely positive contractions $\sigma: A \rightarrow B$ 
and $\tau: B \rightarrow A$ such that

$$
\begin{aligned}
\|x-\tau \sigma(x)\| & <\epsilon, & & x \in \mathscr{F}, \\
\|\sigma(x y)-\sigma(x) \sigma(y)\| & <\epsilon, & & x, y \in \mathscr{F}, \\
\left\|\sigma \alpha_{t}-\beta_{t} \sigma\right\| & <\epsilon, & & t \in[-1,1] .
\end{aligned}
$$

Proof. Since $A$ is nuclear and quasi-diagonal, for any finite subset $\mathscr{F}$ and $\epsilon>0$ there is a triple $(B, \sigma, \tau)$ which satisfies the first two conditions in the lemma (see (iv) of Theorem 5.2.2 of [1]). Though this $\sigma$ has nothing to do with $\alpha$, one can approximate $\sigma$ by a CP contraction $\sigma^{\prime}: A \rightarrow B$ which is $\alpha$-covariant, i.e., the representation of $A$ induced by $\sigma^{\prime}$ is $\alpha$-covariant. More specifically we take a large $\gamma>0$ such that

$$
\frac{\gamma}{2} \int e^{-\gamma|t|}\left\|\alpha_{t}(x)-x\right\| d t \approx 0, \quad x \in \mathscr{F} \cup(\mathscr{F} \cdot \mathscr{F})
$$

where $\mathscr{F} \cdot \mathscr{F}=\{x y \mid x, y \in \mathscr{F}\}$ and set

$$
\sigma^{\prime}=\frac{\gamma}{2} \int e^{-\gamma|t|} \sigma \alpha_{t} d t
$$

Then it follows that $\sigma^{\prime}$ is a CP contraction of $A$ into $B$ such that $\| \sigma(x)-$ $\sigma^{\prime}(x) \| \approx 0$ for $x \in \mathscr{F} \cup(\mathscr{F} \cdot \mathscr{F})$. Thus one may assume that $\sigma^{\prime}$ also satisfies the first two conditions. Note $\sigma^{\prime}$ has the following property: $\sigma^{\prime} \alpha_{s} \leq e^{\gamma|s|} \sigma^{\prime}$, i.e., $e^{\gamma|s|} \sigma^{\prime}-\sigma^{\prime} \alpha_{s}$ is $\mathrm{CP}$, which implies that $\sigma^{\prime}$ is $\alpha$-covariant. This fact follows from Lemma 4.2 below, a version of Stinespring's theorem.

Assume that $B$ acts on a finite-dimensional Hilbert space $\mathscr{H}_{B}$ such that the commutant of $B$ is abelian. There is a covariant representation $(\pi, U)$ and an isometry $V$ from $\mathscr{H}_{B}$ into $\mathscr{H}_{\pi}$ such that $\sigma^{\prime}(x)=V^{*} \pi(x) V$ for $x \in A$. By adding another covariant representation to $(\pi, U)$ we may suppose that $\pi \times U$ is a faithful representation of $A \times_{\alpha}$ R. Since $\alpha$ is quasi-diagonal it follows from Theorem 1.4 of [11] that $(\pi(A), U)$ is quasi-diagonal. Hence there is a finiterank projection $F$ on $\mathscr{H}_{\pi}$ such that $F \geq V V^{*},[F, \pi(x)] \approx 0$ for $x \in \mathscr{F}$ and $\left\|\left[F, U_{t}\right]\right\| \approx 0$ for $t \in[-1,1]$. By Lemma 3.2 applied to the compact operators $\mathscr{K}\left(\mathscr{H}_{\pi}\right)$ and $F \in \mathscr{K}\left(\mathscr{H}_{\pi}\right)$ there is an $\mathrm{Ad} U$-cocycle $Z$ in $\mathscr{K}(\mathscr{H})+\mathrm{C} 1 \subset \mathscr{B}\left(\mathscr{H}_{\pi}\right)$ such that $Z_{t} \approx 1$ for $t \in[-1,1]$ and $\left[F, Z_{t} U_{t}\right]=0$. Define $B_{1}=F \mathscr{B}\left(\mathscr{H}_{\pi}\right) F$ and $\beta_{t}=\operatorname{Ad}\left(Z_{t} U_{t}\right)$ on $B_{1}$ and let $\sigma_{1}=F \pi(\cdot) F$, a CP contraction from $A$ to $B_{1}$. Then since $\left(\beta_{t} \sigma_{1}-\sigma_{1} \alpha_{t}\right)(x)=F\left\{\left(\operatorname{Ad}\left(Z_{t} U_{t}\right)-\operatorname{Ad}\left(U_{t}\right)\right) \pi(x)\right\} F$ for $x \in A$, we have that $\left\|\beta_{t} \sigma_{1}-\sigma_{1} \alpha_{t}\right\| \approx 0$ for $t \in[-1,1]$. Note also that $\sigma_{1}(x y)=F \pi(x y) F \approx F \pi(x) F \pi(y) F=\sigma_{1}(x) \sigma_{1}(y)$ for $x, y \in \mathscr{F}$. Let $\tau_{1}(T)=\tau P_{B}\left(V^{*} T V\right), T \in B_{1}$, where $P_{B}$ is a norm-one projection from 
$\mathscr{B}\left(\mathscr{H}_{B}\right)$ onto $B$. Then $\tau_{1} \sigma_{1}(x)=\tau P_{B}\left(V^{*} F \pi(x) F V\right)=\tau \sigma^{\prime}(x) \approx \tau \sigma(x)$ for $x \in \mathscr{F}$. Thus one can conclude that $\left(B_{1}, \beta, \sigma_{1}, \tau_{1}\right)$ has the required properties.

The following is taken from Section 4 of [11] (see also the proof of Proposition 2 of [10]).

Lemma 4.2. Let $\alpha$ be a flow on a $C^{*}$-algebra $A$ and let $B$ be a $C^{*}$-algebra acting on $\mathscr{H}_{B}$ and $Z$ a unitary flow on $\mathscr{H}_{B}$ such that $t \mapsto \operatorname{Ad} Z_{t}$ defines a flow on $B$. Let $\psi$ be a CP contraction from $A$ into $B$ and $\gamma>0$ such that Ad $Z_{-t} \psi \alpha_{t} \leq e^{\gamma|t|} \psi$ for $t \in \mathrm{R}$. Let $(\pi, V)$ denote the Stinespring pair for $\psi$, i.e., $\pi$ is a representation of $A$ and $V$ is an isometry from $\mathscr{H}_{B}$ into $\mathscr{H}_{\pi}$ such that $\psi(x)=V^{*} \pi(x) V, x \in A$ and $P \mathscr{H}_{\pi}$ is cyclic for $\pi(A)$ with $P=V V^{*}$. Then there is a unitary flow $U=e^{i t H}$ on $\mathscr{H}_{\pi}$ such that $\operatorname{Ad} U_{t} \pi=\pi \alpha_{t}$ and $\|[H, P]\| \leq \gamma / 2$.

Proof. We replace $A$ by the unitization of $A$ and assume $\psi(1)=1$. On the algebraic tensor product $A \otimes \mathscr{H}_{B}$ we define a quasi-inner product by

$$
\langle x \otimes \xi, y \otimes \eta\rangle=\left\langle\psi\left(y^{*} x\right) \xi, \eta\right\rangle_{\mathscr{H}_{B}},
$$

and a representation $\pi$ of $A$ by

$$
\pi(a) x \otimes \xi=a x \otimes \xi .
$$

We define a linear map $V$ from $\mathscr{H}_{B}$ into $A \otimes \mathscr{H}_{B}$ by $V \xi=1 \otimes \xi$. Then we obtain the pair $(\pi, V)$ in the statement by the usual procedure.

We define a linear operator $W_{t}$ on $A \otimes \mathscr{H}_{B}$ by

$$
W_{t} x \otimes \xi=\alpha_{t}(x) \otimes Z_{t} \xi .
$$

We compute for a finite $\operatorname{sum} \zeta=\sum_{i} x_{i} \otimes \xi_{i}$

$$
\begin{aligned}
\left\|W_{t} \zeta\right\|^{2} & =\sum_{i, j}\left\langle\psi \alpha_{t}\left(x_{i}^{*} x_{j}\right) Z_{t} \xi_{j}, Z_{t} \xi_{i}\right\rangle \\
& \leq e^{\gamma|t|} \sum_{i, j}\left\langle\psi\left(x_{i}^{*} x_{j}\right) \xi_{j}, \xi_{i}\right\rangle \\
& =e^{\gamma|t|}\|\zeta\|^{2} .
\end{aligned}
$$

This implies that $W_{t}$ is a well-defined bounded operator in $\mathscr{H}_{\pi}$ such that $\left(W_{t}\right)^{*} W_{t} \leq e^{\gamma|t|} 1$. Moreover the family $W_{t}, t \in \mathrm{R}$ satisfies that $W_{s} W_{t}=W_{s+t}$, $W_{0}=1, t \mapsto W_{t}$ is strongly continuous, and $W_{t} \pi(x)=\pi \alpha_{t}(x) W_{t}, x \in A$. 
Let $W_{t}=e^{i L t}$, i.e., $i L$ is the generator of $W$. Since $\left(W_{t}\right)^{*} W_{t} \leq e^{\gamma|t|}$ it follows that for any $\xi \in D(L)$

$$
\frac{\left\|W_{t} \xi\right\|^{2}-\|\xi\|^{2}}{|t|} \leq \frac{\left(e^{\gamma|t|}-1\right)\|\xi\|^{2}}{|t|} .
$$

By taking the limits $t \downarrow 0$ and $t \uparrow 0$ we derive

$$
-\gamma\|\xi\|^{2} \leq\langle i L \xi, \xi\rangle+\langle\xi, i L \xi\rangle \leq \gamma\|\xi\|^{2},
$$

which implies that $\mathscr{D}\left(L^{*}\right) \supset \mathscr{D}(L)$ and $-\gamma 1 \leq i L-i L^{*} \leq \gamma 1$ as a sesquilinear form on $D(L)$. Let $C$ be the closure of $i\left(L-L^{*}\right) / 2$. Then $\|C\| \leq \gamma / 2$ and $C=C^{*}$, and $L+i C$ is a symmetric operator because $L+i C=L-L / 2+$ $L^{*} / 2=\left(L+L^{*}\right) / 2$ on $\mathscr{D}(L)$. Since $L+i C$ generates a strongly continuous one-parameter group of bounded operators, $L+i C$ must be self-adjoint with $D\left(L^{*}\right)=D(L)$.

Since $W_{t} \pi(x) W_{-t}=\pi \alpha_{t}(x), x \in A$, it follows that $\left(W_{t}\right)^{*} W_{t} \in \pi(A)^{\prime}$ and hence $C \in \pi(A)^{\prime}$. Let $U_{t}=e^{i(L+i C) t}$, which is a unitary flow implementing $\alpha$. We assert that $H=L+i C$ has the required property.

By the definition of $W_{t}$ we deduce $W_{t} V=V Z_{t}$, which implies that $W_{t} P=$ $V Z_{t} V^{*}$ is a unitary on $P \mathscr{H}_{\pi}$ with $P=V V^{*}$. Hence $W_{t} P W_{t}^{*}=P$. Since $\left(W_{t}-1\right) P W_{t}^{*}+P\left(W_{t}^{*}-1\right)=W_{t} P W_{t}^{*}-P=0$, it follows that $L P-P L^{*}=0$ on $D(L)$. Using $H=L+i C=L^{*}-i C$ we deduce that $[H, P]=(L+$ iC) $P-P\left(L^{*}-i C\right)=i(C P+P C)$ on $D(L)$. Namely $[H, P]$ is bounded by $\|C P+P C\|$. On the other hand $P W_{t}^{*} W_{t} P=P$, which implies $P C P=0$. Hence $\|C P+P C\|=\|(1-P) C P\| \leq \gamma / 2$. This completes the proof.

We prepare three technical lemmas which can be derived by using standard techniques which may be found in [4].

Lemma 4.3. There exists a constant $C>0$ satisfying: Let $\gamma$ be a flow on $a C^{*}$-algebra $A$ and let $\delta_{\gamma}$ be the generator of $\gamma$. If $x \in D\left(\delta_{\gamma}\right)$ is such that $\operatorname{Sp}\left(x^{*} x\right) \subset\{0\} \cup[1 / 2,1]$ then the partial isometry $w$ obtained from the polar decomposition of $x$ belongs to $D\left(\delta_{\gamma}\right)$ and satisfies that $\left\|\delta_{\gamma}(w)\right\| \leq C\left\|\delta_{\gamma}(x)\right\|$.

Proof. Let $f$ be a $C^{\infty}$-function on $\mathrm{R}$ with compact support such that $f(0)=0$ and $f(t)=t^{-1 / 2}, t \in[1 / 2,1]$. Then $w$ is obtained as $x f\left(x^{*} x\right)$. We use the formula:

$$
f\left(x^{*} x\right)=\int \hat{f}(t) e^{i t x^{*} x} d t
$$

where $\hat{f}(t)=1 / 2 \pi \int f(s) e^{-i s t} d s$, to derive $f\left(x^{*} x\right) \in D\left(\delta_{\gamma}\right)$ and

$$
\left\|\delta_{\gamma}\left(f\left(x^{*} x\right)\right)\right\| \leq \int|t \hat{f}(t)| d t\left\|\delta_{\gamma}\left(x^{*} x\right)\right\| .
$$


Thus $C=\sqrt{2}+2 \int|t \hat{f}(t)| d t$ will do. See Section 3.2.2 of [4] for details.

Lemma 4.4. There exists a constant $C>0$ satisfying: Let $\gamma$ be a flow on a $C^{*}$-algebra A. Let $p \in A$ be a projection in $D\left(\delta_{\gamma}\right)$ such that $\delta_{\gamma}(p)=0$ and let $e \in D\left(\delta_{\gamma}\right)$ be a projection such that $\|p e-p\| \leq 1 / 8$. Then there is a projection $e^{\prime} \in D\left(\delta_{\gamma}\right)$ such that $p e^{\prime}=p,\left\|e-e^{\prime}\right\| \leq 12\|p e-p\|$, and $\left\|\delta_{\gamma}\left(e^{\prime}\right)\right\| \leq C\left\|\delta_{\gamma}(e)\right\|$.

Proof. Since $\|p e p-p\|=\|p(e p-p)\|<1 / 8$, it follows that $\operatorname{Sp}($ epe $)=$ $\{0\} \cup[7 / 8,1]$. Let $w$ be the partial isometry obtained from the polar decomposition of $p e$. Note that $\|w-p\| \leq\|w-w|p e|\|+\|p e-p\| \leq 2\|p e-p\|$. Note also, from Lemma 4.3, that $\left\|\delta_{\gamma}(w)\right\| \leq C\left\|\delta_{\gamma}(e)\right\|$, where $C$ is the universal constant there. Since $\left\|(1-p)\left(1-w^{*} w\right)(1-p)-(1-p)\right\| \leq$ $\left\|\left(1-w^{*} w\right)(1-p)-(1-p)\right\|=\left\|w^{*} w-w^{*} w p\right\|$ and $\left\|w^{*} w-p\right\| \leq$ $\left\|w^{*}(w-p)\right\|+\left\|\left(w^{*}-p\right) p\right\| \leq 2\|w-p\| \leq 4\|p e-p\|$, it follows that the spectrum of $\left(1-w^{*} w\right)(1-p)\left(1-w w^{*}\right)$ is contained in $\{0\} \cup[1 / 2,1]$. Let $w^{\prime}$ be the partial isometry obtained from $(1-p)\left(1-w^{*} w\right)$ (in $A+\mathrm{C} 1$ if $A$ is not unital). Then $\left\|w^{\prime}-(1-p)\right\| \leq 2\left\|(1-p)\left(1-w^{*} w\right)-(1-p)\right\| \leq$ $2\left\|w^{*} w-p w^{*} w\right\|=\left\|(1-p) w^{*} w\right\| \leq 4\|p e-p\|$. From Lemma 4.3 it follows that $\left\|\delta_{\gamma}\left(w^{\prime}\right)\right\| \leq C\left\|\delta_{\gamma}\left(w^{*} w\right)\right\| \leq 2 C\left\|\delta_{\gamma}(w)\right\|$. We set $u=w+w^{\prime}$, which is a unitary such that $\|u-1\| \leq\|w-p\|+\left\|w^{\prime}-(1-p)\right\| \leq$ $6\|p e-p\|$ and $\left\|\delta_{\gamma}(u)\right\| \leq\left\|\delta_{\gamma}(w)\right\|+\left\|\delta_{\gamma}\left(w^{\prime}\right)\right\| \leq\left(2 C^{2}+C\right)\left\|\delta_{\gamma}(e)\right\|$. We set $e^{\prime}=u e u^{*}$, which is a projection such that $e^{\prime} \geq w e w^{*}=w w^{*}=p$. Note that $\left\|e^{\prime}-e\right\|=\left\|u e u^{*}-e\right\| \leq 2\|u-1\| \leq 12\|p e-p\|$ and that $\left\|\delta_{\gamma}\left(e^{\prime}\right)\right\| \leq 2\left\|\delta_{\gamma}(u)\right\|+\left\|\delta_{\gamma}(e)\right\| \leq\left(4 C^{2}+2 C+1\right)\left\|\delta_{\gamma}(e)\right\|$. This completes the proof.

LEMMA 4.5. Let $\mathscr{K}=\mathscr{K}(\mathscr{H})$ be the compact operators on a Hilbert space $\mathscr{H}$ and $H$ a self-adjoint operator on $\mathscr{H}$ which defines aflow $\gamma: t \mapsto \operatorname{Ad} e^{i t H}$ on $\mathscr{K}$. Then the domain $D\left(\delta_{\gamma}\right)$ is the set of operators $x \in \mathscr{K}$ such that $x D(H) \subset$ $D(H)$ and $[i H, x]$ on $D(H)$ extends to a compact operator, which is $\delta_{\gamma}(x)$. If $x \in \mathscr{K}$ is of finite rank and $x D(H) \subset D(H)$ and $[i H, x]$ is bounded on $D(H)$ then the closure of $[i H, x]$ is compact and thus $x \in D\left(\delta_{\gamma}\right)$ and $\delta_{\gamma}(x)=\overline{[i H, x]}$.

Proof. Let $\bar{\gamma}_{t}=\operatorname{Ad} e^{i t H}$ on the bounded operators $B(\mathscr{H})$. Then $\bar{\gamma}$ is a oneparameter group of automorphisms of the type I factor $B(\mathscr{H})$ and $t \mapsto \bar{\gamma}(Q)$ is continuous in the strong operator topology for $Q \in B(\mathscr{H})$. Let $L$ be the generator of $\bar{\gamma}$. Then $D(L)$ consists of $Q \in B(\mathscr{H})$ such that $Q D(H) \subset D(H)$ and $[i H, Q]$ is bounded on $D(H)$ and if $Q \in D(L)$ then $L(Q)$ is the closure of [iH,Q]. (See Proposition 3.2.55 of [4].) Thus if $x \in D\left(\delta_{\gamma}\right)$ then it follows that $x D(H) \subset D(H)$ and $[i H, x]$ on $D(H)$ extends to a compact operator. Conversely if $x \in \mathscr{K}$ satisfies the latter conditions, then it follows that $t \mapsto$ 
$i H e^{i t H} x e^{-i t H} \xi$ is continuous for $\xi \in D(H)$. Hence if $f \in L^{1}(\mathrm{R})$ is such that $\hat{f}$ has compact support then the closure of $\left[i H, \gamma_{f}(x)\right]$ is equal to

$$
\int f(t) e^{i t H} \overline{[i H, x]} e^{-i t H} d t,
$$

where $\gamma_{f}(x)=\int f(t) \gamma_{t}(x) d t$ belongs to $D\left(\delta_{\gamma}\right)$ as having compact $\gamma$-spectrum. Since $\overline{\left[i H, \gamma_{f}(x)\right]}=\delta_{\gamma}\left(\gamma_{f}(x)\right)$ and $\delta_{\gamma}$ is closed it follows that $x \in D\left(\delta_{\gamma}\right)$ and $\delta_{\gamma}(x)=\overline{[i H, x]}$.

Let $x \in \mathscr{K}$ be of finite rank. Since the range $V$ of $x$ is finite-dimensional and contained in $D(H)$ it follows that $H \mid V$ is bounded. If $\left(\xi_{n}\right)$ is a bounded sequence in $D(H)$ then there is a subsequence $\left(\xi_{n}^{\prime}\right)$ of $\left(\xi_{n}\right)$ such that $x \xi_{n}^{\prime}$ converges; so $i H x \xi_{n}^{\prime}$ converges. Since $\left(x i H \xi_{n}^{\prime}\right)$ is a bounded sequence in $V$ we can choose a subsequence $\left(\xi_{n}^{\prime \prime}\right)$ of $\left(\xi_{n}^{\prime}\right)$ such that $x i H \xi_{n}^{\prime \prime}$ converges. Thus $[i H, x] \xi_{n}^{\prime \prime}$ converges and $\overline{[i H, x]}$ is compact. By the way in general we have to require $[i H, x]$ to be compact (not just bounded) to ensure $x \in D\left(\delta_{\gamma}\right)$.

We will apply Lemma 4.4 to the situation described in Lemma 4.5 in the proof of the following lemma.

Lemma 4.6. Let $B$ be a separable nuclear $C^{*}$-algebra on a Hilbert space $\mathscr{H}$ and $U$ a unitary flow on $\mathscr{H}$ such that $B \supset \mathscr{K}(\mathscr{H}), t \mapsto \operatorname{Ad} U_{t}(x)$ defines a norm-continuous flow on $B$. Let $\alpha$ denote the flow on $A=B / \mathscr{K}(\mathscr{H})$ induced by $t \mapsto \operatorname{Ad} U_{t} \mid B$. Then if $(B, U)$ is quasi-diagonal then $(A, \alpha)$ is quasi-diagonal.

Proof. Under the assumption we shall prove the condition (2) of Theorem 1.5 of [11]. Namely for any finite subset $\mathscr{F}$ of $A$ and $\epsilon>0$ we shall construct a finite-dimensional $C^{*}$-algebra $D$, a flow $\beta$ on $D$, and a CP map $\phi$ of $A$ into $D$ such that $\|\phi\| \leq 1,\|\phi(x)\| \geq(1-\epsilon)\|x\|$ and $\|\phi(x) \phi(y)-\phi(x y)\| \leq \epsilon\|x\|\|y\|$ for $x, y \in \mathscr{F}$, and $\left\|\beta_{t} \phi-\phi \alpha_{t}\right\|<\epsilon$ for $t \in[-1,1]$.

Since $(B, U)$ is quasi-diagonal there is an increasing sequence $\left(P_{n}\right)$ of finiterank projections on $\mathscr{H}$ such that $\lim _{n} P_{n}=1,\left\|\left[P_{n}, a\right]\right\| \rightarrow 0$ for all $a \in B$, and $\left\|\left[P_{n}, H\right]\right\|<2^{-n}$ where $H$ is the self-adjoint generator of $U$. Note that the last condition means that $P_{n} \mathscr{D}(H) \subset \mathscr{D}(H)$ and $\left\|\left[P_{n}, H\right] \mid \mathscr{D}(H)\right\|<2^{-n}$. Let $P_{0}=0$ and let $H_{0}=\sum_{n=1}^{\infty}\left(P_{n}-P_{n-1}\right) H\left(P_{n}-P_{n-1}\right)$, which is a well-defined self-adjoint operator. Since $H-H_{0}$ on $\mathscr{D}(H)$ is compact, we may take the unitary flow generated by $H_{0}$ instead of $U$, which still leaves $B$ invariant and defines a flow on $B$ dropping to the same flow $\alpha$ on the quotient $A=B / \mathscr{K}(\mathscr{H})$. Thus we assume now that $\left[P_{n}, H\right]=0$ for all $n$.

The existence of the above $\left(P_{n}\right)$ follows by the following arguments. Suppose that $P_{n}$ was chosen. We have to define $P_{n+1}$. The main difficulty lies in finding one strictly bigger than $P_{n}$. First let $h=-\left(1-P_{n}\right) H P_{n}-P_{n} H\left(1-P_{n}\right)$, 
which is a compact operator with norm less than or equal to $\left\|\left[H, P_{n}\right]\right\|$. We choose a constant $C>0$ as in Lemma 4.4. Let $\epsilon>0$ be sufficiently small and set $\delta=\epsilon / C$. Then we find a sufficiently large finite-rank projection $E$ such that $\left\|P_{n} E-P_{n}\right\|<\delta,\|h E-h\|<\delta / 4,\|[H, E]\|<\delta / 2$, and $\|[E, a]\|<\delta$ for a finite number of $a \in B$ prescribed. Note that $\left[H+h, P_{n}\right]=0$ and $\|[H+h, E]\| \leq \delta$. By applying Lemma 4.4 to the pair $P_{n}, E$ with the derivation $i[H+h, \cdot]$ on the compact operators, we obtain a finite-rank projection $E^{\prime}$ such that $P_{n} \leq E^{\prime},\left\|E-E^{\prime}\right\|<\epsilon$, and $\left\|\left[H+h, E^{\prime}\right]\right\|<\epsilon$. Since $\left\|\left[h, E^{\prime}\right]\right\| \leq$ $2 \epsilon\|h\|+\|[h, E]\|<2 \epsilon+\delta / 2$, we deduce that $\left\|\left[H, E^{\prime}\right]\right\| \leq\left(3+(2 C)^{-1}\right) \epsilon$. Thus for a sufficiently small $\epsilon>0$ we can set $P_{n+1}=E^{\prime}$.

Since $A$ is nuclear there is a completely positive (CP) contraction $\phi$ of $A$ into $B$ such that $Q \phi=\mathrm{id}$, where $Q$ is the quotient map of $B$ onto $A=B / \mathscr{K}(\mathscr{H})$ [5]. Let $\phi_{t}=\operatorname{Ad} U_{-t} \phi \alpha_{t}$ for $t \in \mathrm{R}$, which is also a CP map. Since $Q \phi_{t}=\mathrm{id}$, it follows that $\phi_{t}(a)-\phi(a) \in \mathscr{K}(\mathscr{H})$. Since $t \mapsto \phi_{t}(a)$ is norm-continuous one deduces that $\left\|\left(1-P_{n}\right)\left(\phi_{t}(a)-\phi(a)\right)\left(1-P_{n}\right)\right\| \rightarrow 0$ as $n \rightarrow \infty$ uniformly in $t$ on every compact subset of $\mathrm{R}$ for all $a \in A$.

Let $\mathscr{F}$ be a finite subset of $A$ and $\epsilon>0$. Let

$$
\psi=\frac{\epsilon}{2} \int e^{-\epsilon|t|} \phi_{t} d t
$$

which is a CP map of $A$ into $B$ such that $Q \psi=$ id. Since $e^{-\epsilon|t|} \psi \leq \operatorname{Ad} U_{-t} \psi \alpha_{t}$ $\leq e^{\epsilon|t|} \psi$ it follows that $\left\|\psi \alpha_{t}-\operatorname{Ad} U_{t} \psi\right\| \leq e^{\epsilon|t|}-1$.

Since $\psi(x) \psi(y)-\psi(x y) \in \mathscr{K}(\mathscr{H})$ there is an $N \in \mathrm{N}$ such that $\|(1-$ $\left.P_{N}\right)(\psi(x) \psi(y)-\psi(x y))\left(1-P_{N}\right)\|<\epsilon\| x\|\| y \| / 2$ for $x, y \in \mathscr{F}$. There exists an $n \geq N$ such that for any $m \geq n\left\|\left[P_{m}, \psi(x)\right]\right\|<\epsilon / 4$ for $x \in \mathscr{F}$. Since $Q \psi=$ id we have that $\left\|\left(1-P_{n}\right) \psi(x)\left(1-P_{n}\right)\right\| \geq\|x\|$ for $x \in A$. We then choose $m>n$ such that $\left\|\left(P_{m}-P_{n}\right) \psi(x)\left(P_{m}-P_{n}\right)\right\| \geq(1-\epsilon)\|x\|$ for $x \in \mathscr{F}$. Let $E=P_{m}-P_{n}$. Since $\|[E, \psi(x)]\| \leq \epsilon\|x\| / 2$ for $x \in \mathscr{F}$, we obtain that $\|E \psi(x) E \psi(y) E-E \psi(x y) E\| \leq \epsilon\|x\|\|y\| / 2+\| E \psi(x) \psi(y) E-$ $E \psi(x y) E\|\leq \epsilon\| x\|\| y \|$. By setting $D=E \mathscr{B}(\mathscr{H}) E, \beta_{t}=\operatorname{Ad} U_{t} \mid D$, and $\phi(x)=E \psi(x) E, x \in A$, we obtain the desired triple $(D, \beta, \phi)$.

The following result is proved by mimicking the proof of Theorem 5.2.2 of [1].

THEOREM 4.7. Let $\alpha$ be a flow on a separable $C^{*}$-algebra. Then the following conditions are equivalent:

(1) $(A, \alpha)$ is obtained as the inductive limit of $a *$-linear generalized inductive system of flows on finite-dimensional $C^{*}$-algebras where the coherent maps are all completely positive contractions.

(2) A is nuclear and $\alpha$ is an MF flow. 
(3) $A$ is nuclear and there is an essential quasi-diagonal extension $B$ of $A$ by the compact operators $\mathscr{K}$ and a unitary flow $U \in M(\mathscr{K})$ such that $t \mapsto \operatorname{Ad} U_{t}$ defines a flow on $B,(B, U)$ is quasi-diagonal, and $Q \operatorname{Ad} U_{t}=\alpha_{t} Q$, where $Q$ is the quotient map of $B$ onto $A$.

(4) A is nuclear and $\alpha$ is quasi-diagonal.

(5) For any finite subset $\mathscr{F}$ of $A$ and $\epsilon>0$ there is a flow $\beta$ on a finitedimensional $C^{*}$-algebra $B$ and completely positive contractions $\sigma$ : $A \rightarrow B$ and $\tau: B \rightarrow A$ such that

$$
\begin{aligned}
\|x-\tau \sigma(x)\| & <\epsilon, & & x \in \mathscr{F}, \\
\|\sigma(x y)-\sigma(x) \sigma(y)\| & <\epsilon, & & x, y \in \mathscr{F}, \\
\left\|\sigma \alpha_{t}-\beta_{t} \sigma\right\| & <\epsilon, & & t \in[-1,1] .
\end{aligned}
$$

(6) A is nuclear and there is a continuous field of flows $\left(A_{n}, \alpha_{n}\right)$ over $\mathrm{N} \cup\{\infty\}$ such that $A_{n}$ is finite-dimensional for $n \in \mathrm{N}$ and $\left(A_{\infty}, \alpha_{\infty}\right) \cong(A, \alpha)$.

(7) $A$ is nuclear and there is a continuous field of flows $\left(A_{n}, \alpha_{n}\right)$ over $\mathrm{N} \cup\{\infty\}$ such that $A_{n} \cong M_{k_{n}}$ for some $k_{n}$ for $n \in \mathrm{N}$ and $\left(A_{\infty}, \alpha_{\infty}\right) \cong(A, \alpha)$.

Proof. (1) $\Rightarrow$ (2): That $A$ is nuclear follows from Proposition 5.1 .3 of [1] and that $\alpha$ is an MF flow follows from Theorem 3.10.

(2) $\Rightarrow(3)$ : This follows from (2) $\Rightarrow$ (3) of Theorem 3.10.

$(3) \Rightarrow(4)$ : This follows from Lemma 4.6.

(4) $\Rightarrow$ (5): This follows from Lemma 4.1.

The equivalences between (2), (6), and (7) follow from those between (2), (4), and (5) in Theorem 3.10.

It remains to prove $(5) \Rightarrow(1)$. We define a sequence $\left(A_{n}, \alpha_{n}\right)$ of flows on finite-dimensional $C^{*}$-algebras and sequences of CP contractions $\sigma_{n}: A \rightarrow$ $A_{n}$ and $\tau_{n}: A_{n} \rightarrow A$ as follows. Let $\left(x_{n}\right)$ be a dense sequence in $A$. We choose $\left(A_{1}, \alpha_{1}\right)$ and $\mathrm{CP}$ contractions $\sigma_{1}: A \rightarrow A_{1}$ and $\tau_{1}: A_{1} \rightarrow A$ such that $\left\|x_{1}-\tau_{1} \sigma_{1}\left(x_{1}\right)\right\|<1 / 2$ and $\left\|\sigma_{1} \alpha_{t}-\alpha_{1, t} \sigma_{1}\right\|<1 / 2$ for $t \in[-1,1]$. Suppose that $\left(A_{m}, \alpha_{m}, \sigma_{m}, \tau_{m}\right)$ is defined up to $m=n$. Let $N \in \mathrm{N}$ be such that if $|t|<1 / N$ then $\left\|\alpha_{t}(x)-x\right\|<2^{-n}$ for all $x$ in the unit ball of $\tau_{n}\left(A_{n}\right)$. Let $V_{n}$ be the finite-dimensional subspace generated by $\alpha_{k / N}(x)$ with $x \in \tau_{n}\left(A_{n}\right)$, $k=0, \pm 1, \ldots, \pm N$, and $x y$ with $x, y \in \tau_{n}\left(A_{n}\right)$ and $V_{n-1} \cup\left\{x_{n}\right\}$. We choose $\left(A_{n+1}, \alpha_{n+1}, \sigma_{n+1}, \tau_{n+1}\right)$ such that

$$
\begin{aligned}
\left\|x-\tau_{n+1} \sigma_{n+1}(x)\right\| & \leq 2^{-n-1}\|x\|, & & x \in V_{n}, \\
\left\|\sigma_{n+1}(x) \sigma_{n+1}(y)-\sigma_{n+1}(x y)\right\| & \leq 2^{-n-1}\|x\|\|y\|, & & x, y \in V_{n}, \\
\left\|\sigma_{n+1} \alpha_{t}-\alpha_{n+1, t} \sigma_{n+1}\right\| & <2^{-n-1}, & & t \in[-1,1] .
\end{aligned}
$$

Note that $\left(V_{n}\right)$ is increasing with dense union in $A$. 
Let $\phi_{n}=\sigma_{n+1} \tau_{n}: A_{n} \rightarrow A_{n+1}$, a CP contraction. We can show that $\phi_{n}$ is almost multiplicative on $\phi_{n-1}\left(A_{n-1}\right)$ as follows. If $x \in A_{n-1}$, then $\phi_{n}\left(\phi_{n-1}(x) \phi_{n-1}(x)\right)$ is approximately equal to $\sigma_{n+1} \tau_{n} \sigma_{n}\left(\tau_{n-1}(x) \tau_{n-1}(y)\right)$ (since $\sigma_{n}$ is approximately multiplicative) and then to

$$
\sigma_{n+1}\left(\tau_{n-1}(x) \tau_{n-1}(y)\right) \approx \sigma_{n+1} \tau_{n-1}(x) \sigma_{n+1} \tau_{n-1}(y) \approx \phi_{n} \phi_{n-1}(x) \phi_{n} \phi_{n-1}(y),
$$

where the error is up to $5 \cdot 2^{-n}\|x\|\|y\|$. We can show that $\alpha_{n+1, t} \phi_{n}-\phi_{n} \alpha_{n, t}$ is almost equal to zero on $\phi_{n-1}\left(A_{n-1}\right)$. If $x \in A_{n-1}$ and $t \in[-1,1]$, then $\left(\alpha_{n+1, t} \phi_{n}-\phi_{n} \alpha_{n, t}\right) \phi_{n-1}(x)=\left(\alpha_{n+1, t} \sigma_{n+1} \tau_{n}-\sigma_{n+1} \tau_{n} \alpha_{n, t}\right) \sigma_{n} \tau_{n-1}(x)$ is approximately equal to

$$
\begin{aligned}
\sigma_{n+1} \alpha_{t} \tau_{n} \sigma_{n} \tau_{n-1}(x)-\sigma_{n+1} \tau_{n} \sigma_{n} \alpha_{t} \tau_{n-1}(x) & \\
& \approx \sigma_{n+1} \alpha_{t} \tau_{n-1}(x)-\sigma_{n+1} \alpha_{t} \tau_{n-1}(x)=0,
\end{aligned}
$$

where the error is up to $6 \cdot 2^{-n}\|x\|$.

Now we have the following commutative diagram:

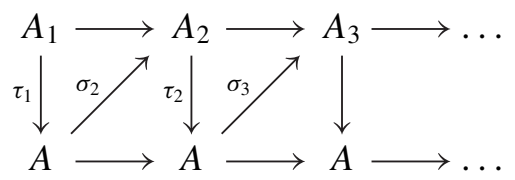

where the arrows represent $\mathrm{CP}$ contractions. Hence the upper sequence and the lower sequence define the same object as Banach spaces (at least). Let

$$
\psi_{m, n}=\tau_{n-1} \sigma_{n-1} \tau_{n-2} \sigma_{n-2} \cdots \tau_{m+1} \sigma_{m+1}
$$

for $n>m$, a CP contraction from the $m$ 'th $A$ into $n$ 'th $A$. Since $\left(\psi_{n, m}(x)\right)_{n \geq m}$ is a Cauchy sequence for each $x \in A$ we denote the limit by $\Psi_{m}(x)$. Then $\left(\Psi_{n}\right)$ defines a sequence of CP contractions from $A$ into $A$ and satisfies $\Psi_{n} \psi_{m, n}=$ $\Psi_{m}$ for $n \geq m$. Since $\bigcup_{n} \Psi_{n}(A)$ is dense in $A$ it follows that the lower sequence defines $A$ as a Banach space. From the way to define product in the inductive limit, one concludes that the lower sequence defines $A$ as a $C^{*}$-algebra. Since $\Psi_{n} \alpha_{t} \psi_{m, n}(x)$ converges to $\alpha_{t} \Psi_{m}(x)$ as $n \rightarrow \infty$, the lower sequence defines $(A, \alpha)$ as a flow. Then one argues the upper sequence defines $(A, \alpha)$ as well.

We will call $\alpha$ an NF flow if it satisfies the conditions described in the above theorem. Since quasi-diagonality is preserved under cocycle perturbations (2.2 of [4]), a cocycle perturbation of an NF flow is also an NF flow. 


\section{Strongly quasi-diagonal flows}

Definition 5.1. Let $A$ be a $C^{*}$-algebra and let $\alpha$ be a flow on $A$. We call $\alpha$ strongly quasi-diagonal if $(\pi(A), U)$ is quasi-diagonal for any covariant representation $(\pi, U)$.

Note that the $C^{*}$-algebra $A$ is called strongly quasi-diagonal if $\pi(A)$ is quasi-diagonal for any representation $\pi$ of $A$.

A quasi-diagonal flow need not be strongly quasi-diagonal. If $\alpha$ is an arbitrary flow on a quasi-diagonal $C^{*}$-algebra $A$, the flow $\beta$ on $B=A \otimes C[0,1]$ defined by $\beta_{t}(x)(s)=\alpha_{s t}(x(s))$ is quasi-diagonal and has $(A, \alpha)$ as a quotient (see Proposition 2.15 of [11]). Hence if $(A, \alpha)$ is not quasi-diagonal then $(B, \beta)$ is not strongly quasi-diagonal.

In a similar fashion we can define a notion of strong pseudo-diagonality. Then it follows that an approximately inner flow on a quasi-diagonal $C^{*}$ algebra is strongly pseudo-diagonal (see the proof of Proposition 2.17 of [11]). But we do not know if they are strongly quasi-diagonal or not.

The following shows the above definition is not empty.

Lemma 5.2. Let A be a strongly quasi-diagonal $C^{*}$-algebra. Then the trivial flow $\alpha=\mathrm{id}$ is strongly quasi-diagonal.

Proof. Let $(\pi, U)$ be a covariant representation of $(A, \alpha)$, i.e., $U$ is a unitary flow on $\mathscr{H}_{\pi}$ such that $U_{t} \in \pi(A)^{\prime}$. Let $H$ be the self-adjoint generator of $U$ and $E$ the spectral measure of $H$.

Let $\mathscr{F}$ be a finite subset of $A$, let $\mathscr{G}$ be a finite subset of $\mathscr{H}_{\pi}$, and let $\epsilon>0$. We may suppose that all $\xi \in \mathscr{G}$ belong to $E(a, b] \mathscr{H}_{\pi}$ for some $a<b$. Let $\left(a_{i}\right)_{i=0}^{N}$ be an increasing sequence in R such that $a_{0}=a, a_{N}=b$, and $a_{i}-a_{i-1}<\epsilon$ for $i=1,2, \ldots, N$. Let $\mathscr{G}_{i}=\left\{E\left(a_{i-1}, a_{i}\right] \xi \mid \xi \in \mathscr{G}\right\}$. Since $\pi(A) E\left(a_{i-1}, a_{i}\right]$ is quasi-diagonal on the subspace $\mathscr{H}_{i}=E\left(a_{i-1}, a_{i}\right] \mathscr{H}_{\pi}$, there is a finite-rank operator $E_{i}$ on $\mathscr{H}_{i}$ such that $\left\|\left[E_{i}, \pi(x) E\left(a_{i-1}, a_{i}\right]\right]\right\| \leq \epsilon\|x\|$ for $x \in \mathscr{F}$ and $\left\|\left(E\left(a_{i-1}, a_{i}\right]-E_{i}\right) \xi\right\| \leq \epsilon\|\xi\|$ for $\xi \in \mathscr{G}_{i}$. Let $E=\sum_{i=1}^{N} E_{i}$, which is a finite-rank projection on $\mathscr{H}_{\pi}$. Since $[E, \pi(x)]=\sum_{i} E\left(a_{i-1}, a_{i}\right]\left[E_{i}, \pi(x)\right]$, we deduce that

$$
\|[E, \pi(x)]\|=\max _{i}\left\|E\left(a_{i-1}, a_{i}\right]\left[E_{i}, \pi(x)\right]\right\| \leq \epsilon\|x\|
$$

for $x \in \mathscr{F}$. Since $(1-E) \xi=\sum_{i}\left(E\left(a_{i-1}, a_{i}\right]-E_{i}\right) E\left(a_{i-1}, a_{i}\right] \xi$, we deduce that

$$
\|(1-E) \xi\|^{2}=\sum_{i}\left\|\left(E\left(a_{i-1}, a_{i}\right]-E_{i}\right) E\left(a_{i-1}, a_{i}\right] \xi\right\|^{2} \leq \epsilon^{2}\|\xi\|^{2}
$$


for $\xi \in \mathscr{G}$. Since $U_{t} E U_{t}^{*}-E=\sum_{i} E\left(a_{i-1}, a_{i}\right]\left(U_{t} E_{i} U_{t}^{*}-E_{i}\right)$ we deduce that

$$
\left\|U_{t} E U_{t}^{*}-E\right\|=\max _{i}\left\|U_{t} E_{i} U_{t}^{*}-E_{i}\right\| \leq \epsilon|t| .
$$

This shows that $(\pi(A), U)$ is quasi-diagonal.

Proposition 5.3. Let $\alpha$ be a strongly quasi-diagonal flow on $A$ and let $u$ be an $\alpha$-cocycle. Then $\operatorname{Ad} u \alpha$ is also strongly quasi-diagonal.

Proof. Let $(\pi, U)$ be a covariant representation of $(A, \operatorname{Ad} u \alpha)$. Then $t \mapsto$ $V_{t}=\pi\left(u_{t}^{*}\right) U_{t}$ is a unitary flow implementing $\alpha$. Hence by assumption $(\pi(A), V)$ is quasi-diagonal. Then it follows from the proof of Proposition 2.2 of [11] that $(\pi(A), U)$ is quasi-diagonal.

Corollary 5.4. Let $\alpha$ be a flow on A. Let $B$ be an $\alpha$-invariant hereditary $C^{*}$-subalgebra of $A$ such that $B$ generates $A$ as a closed ideal. Then $\alpha$ is strongly quasi-diagonal if and only if $\alpha \mid B$ is strongly quasi-diagonal.

Proof. Any covariant representation of $(B, \alpha \mid B)$ extends to a covarint representation of $(A, \alpha)$. Hence if $(A, \alpha)$ is strongly quasi-diagonal then so is $(B, \alpha \mid B)$.

Suppose that $(B, \alpha \mid B)$ is strongly quasi-diagonal. Then $(B \otimes \mathscr{K}, \alpha \mid B \otimes \mathrm{id})$ is also strongly quasi-diagonal, where $\mathscr{K}$ is the $C^{*}$-algebra of compact operators on a separable infinite-dimensional Hilbert space. If $A$ is separable then $(A \otimes$ $\mathscr{K}, \alpha \otimes$ id) is isomorphic to a cocycle perturbation of $(B \otimes \mathscr{K}, \alpha \mid B \otimes$ id). Thus one concludes that $(A, \alpha)$ is strongly quasi-diagonal in this case. One can reduce the general case to this case (see the proof of 2.7 of [11]).

Proposition 5.5. Let A be a $C^{*}$-algebra and let $\alpha$ be a flow on A. Suppose that there is an increasing sequence $\left(A_{n}\right)$ of $\alpha$-invariant $C^{*}$-subalgebras of $A$ with dense union such that $A_{n}$ is strongly quasi-diagonal and the restriction of $\alpha$ to $A_{n}$ is inner, i.e., $\alpha \mid A_{n}=\operatorname{Ad} u_{t}$ for some unitary flow $u$ in $M\left(A_{n}\right)$. Then $\alpha$ is strongly quasi-diagonal.

Proof. Let $(\pi, U)$ be a covariant representation of $(A, \alpha)$. Then by assumption $\left(\pi\left(A_{n}\right), U\right)$ is quasi-diagonal for any $n$. Hence $(\pi(A), U)$ is also quasi-diagonal.

COROLlary 5.6. Any AF flow is strongly quasi-diagonal.

Proof. Let $\alpha$ be an AF flow on $A$. Then $A$ is an $\mathrm{AF} C^{*}$-algebra and there is an increasing sequence $\left(A_{n}\right)$ of finite-dimensional $\alpha$-invariant $C^{*}$-algebras of $A$ with dense union. Since $\alpha \mid A_{n}$ is inner and $A_{n}$ is strongly quasi-diagonal this follows from the above proposition. 
Lemma 5.7. Let $\alpha$ be a flow on a separable $C^{*}$-algebra A. Suppose that there is a sequence $\left(\pi_{i}, U^{i}\right)$ of covariant irreducible representations of $(A, \alpha)$ such that $\bigoplus_{i} \pi_{i}$ is faithful, $\left(\pi_{i}\right)$ are mutually disjoint, and $\left(\pi_{i}(A), U^{i}\right)$ is quasidiagonal for all $i$. Then there is an $\alpha$-cocyle $u$ and an increasing sequence $\left(A_{n}\right)$ of Ad $u \alpha$-invariant residually finite-dimensional $(R F D) C^{*}$-subalgebras of $A$ with dense union such that $\pi_{i} \mid A_{n}$ is equivalent to a direct sum of $\mathrm{Ad} u \alpha-$ covariant finite-dimensional irreducible representations for all $i$ and $n$.

Proof. Let $\left(x_{i}\right)$ be a dense sequence of the unit ball of $A_{s a}=\{x \mid x=$ $\left.x^{*} \in A\right\}$. Let $\mathscr{H}_{i}$ denote the representation Hilbert space for $\pi_{i}$ and $\left(\xi_{k}^{(i)}\right)$ be an orthonormal basis of $\mathscr{H}_{i}$. Let $H_{i}$ denote the self-adjoint generator of $U^{i}$ and $\epsilon>0$.

Let $E_{11}$ be a finite-rank projection on $\mathscr{H}_{1}$ such that $\left\|\left(1-E_{11}\right) \xi_{1}^{(1)}\right\|<\epsilon / 2$, $\left\|\left[E_{11}, \pi_{1}\left(x_{1}\right)\right]\right\|<\epsilon / 2$, and $\left\|\left[E_{11}, H_{1}\right]\right\|<\epsilon / 2$.

Let $E_{11}^{\prime}$ be the range projection of $\left(1-E_{11}\right) x_{1} E_{11}$, which is a finite-rank projection orthogonal to $E_{11}$. We apply Kadison's transitivity theorem to an operator on the finite-dimensional space $\left(E_{11}+E_{11}^{\prime}\right) \mathscr{H}_{1}$ to find a $y_{11} \in A_{s a}$ such that $\left\|y_{11}\right\|=\left\|E_{11}^{\prime} \pi_{1}\left(x_{1}\right) E_{11}\right\|<\epsilon / 2$ and

$$
\pi_{1}\left(y_{11}\right)\left(E_{11}+E_{11}^{\prime}\right)=E_{11}^{\prime} \pi_{1}\left(x_{1}\right) E_{11}+E_{11} \pi_{1}\left(x_{1}\right) E_{11}^{\prime} .
$$

Note that $\left[E_{11}, \pi_{1}\left(x_{1}-y_{11}\right)\right]=0$. Similarly there is an $h_{1} \in A_{s a}$ such that $\left\|h_{1}\right\|<\epsilon / 2$ and $\left[E_{11}, H_{1}-\pi_{1}\left(h_{1}\right)\right]=0$. We set $y_{i 1}=0$ for $i>1$.

Next we find finite-rank projections $E_{12}$ in $\mathscr{H}_{1}$ and $E_{22}$ in $\mathscr{H}_{2}$ such that $E_{11} \leq E_{12},\left\|\left(1-E_{12}\right) \xi_{i}^{(1)}\right\|<\epsilon / 4$ and $\left\|\left(1-E_{22}\right) \xi_{i}^{(2)}\right\|<\epsilon / 4$ for $i=1,2$, $\left\|\left[E_{12}, \pi_{1}\left(x_{i}-y_{i 1}\right)\right]\right\|<\epsilon / 4$ and $\left\|\left[E_{22}, \pi_{2}\left(x_{i}-y_{i 1}\right)\right]\right\|<\epsilon / 4$ for $i=1,2$, and $\left\|\left[E_{12}, H_{1}-\pi_{1}\left(h_{1}\right)\right]\right\|<\epsilon / 4$, and $\left\|\left[E_{22}, H_{2}-\pi_{2}\left(h_{1}\right)\right]\right\|<\epsilon / 4$. (Since $\left[E_{11}, H_{1}-\pi_{1}\left(h_{1}\right)\right]=0$, we can impose the strict inequality $E_{11} \leq E_{12}$ from an approximate one as follows. If $E_{11} \lesssim E_{12}$ let $F$ be the projection obtained from $E_{12} E_{11} E_{12} \approx E_{11}$ by continuous functional calculus and define $X=E_{11} F+\left(1-E_{11}\right)\left(E_{12}-F\right) \approx E_{12}$ and let $X=V E_{12}$ be the polar decomposition of $X$. We take $V E_{12} V^{*}$ (which dominates $E_{11}$ ) instead of $E_{12}$. Since $\left\|\left[F, H_{1}-\pi_{1}\left(h_{1}\right)\right]\right\| \approx 0$ and $\left\|\left[X, H_{1}-\pi_{1}\left(h_{1}\right)\right]\right\| \approx 0$ depending only on $\left\|\left[E_{12}, H_{1}-\pi_{1}\left(h_{1}\right)\right]\right\| \approx 0$, we conclude that $\left\|\left[V E_{12} V^{*}, H_{1}-\pi_{1}\left(h_{1}\right)\right]\right\| \approx 0$.) Let $E_{k 2}^{(i)}$ be the range projection of $\left(1-E_{k 2}\right) \pi_{k}\left(x_{i}-y_{i 1}\right) E_{k 2}$ for $k=1,2$ and $i=1,2$. There is an $y_{i 2} \in A_{s a}$ for $i=1,2$ such that $\left\|y_{i 2}\right\|<\epsilon / 4$ and

$$
\pi_{k}\left(y_{i 2}\right)\left(E_{k 2}+E_{k 2}^{(i)}\right)=E_{k 2}^{(i)} \pi_{k}\left(x_{i}-y_{i 1}\right) E_{k 2}+E_{k 2} \pi_{k}\left(x_{i}-y_{i 1}\right) E_{k 2}^{(i)}
$$

for $k=1,2$, where we have used the fact that $\pi_{1}$ and $\pi_{2}$ are mutually disjoint. Note that $\left[E_{12}, \pi\left(x_{1}-y_{11}-y_{12}\right)\right]=0$ and $\left[E_{12}, \pi\left(x_{2}-y_{21}-y_{22}\right)\right]=0$. Since $\pi\left(y_{12}\right) E_{11}=0$ it also follows that $\left[E_{11}, \pi\left(x_{1}-y_{11}-y_{12}\right)\right]=0$. Similarly 
there is an $h_{2} \in A_{s a}$ such that $\left\|h_{2}\right\|<\epsilon / 4$ and $\left[E_{k 2}, H_{k}-\pi_{k}\left(h_{1}+h_{2}\right)\right]=0$ for $k=1,2$. Note also that $\left[E_{11}, H_{1}-\pi_{1}\left(h_{1}+h_{2}\right)\right]=0$. We set $y_{i 2}=0$ for $i>2$. Note that we have defined $E_{11} \leq E_{12}$ on $\mathscr{H}_{1}$ and $E_{22}$ on $\mathscr{H}_{2}$. We will set $E_{k j}=0$ for $k>j$.

We repeat this process. After $n$ steps we find $y_{i j} \in A_{s a}$ for $1 \leq j \leq n$ and $h_{i} \in A_{s a}$ for $1 \leq i \leq n$ and finite rank projections $E_{k j}$ in $\mathscr{H}_{k}$ for $1 \leq j \leq n$ satisfying the following conditions: $y_{i j}=0$ for $i>j,\left\|y_{i j}\right\|<2^{-j} \epsilon, E_{k j}=0$ for $k>j,\left(E_{k j}\right)_{j}$ is an increasing sequence of finite-rank projections on $\mathscr{H}_{k}$ strongly converging to 1 , and

$$
\left[E_{k j}, \pi_{k}\left(x_{i}-\sum_{m=1}^{n} y_{i m}\right)\right]=0, \quad 1 \leq i \leq j, \quad\left[E_{k j}, H_{k}-\pi_{k}\left(\sum_{m=1}^{n} h_{m}\right)\right]=0
$$

for $k \leq j \leq n$. Thus by setting $y_{i}=x_{i}-\sum_{m=1}^{\infty} y_{i m}$ and $h=\sum_{m=1}^{\infty} h_{m}$ we obtain the following equalities: $\left[E_{k j}, \pi_{k}\left(y_{i}\right)\right]=0$ for $i \leq j,\left[E_{k j}, H_{k}-\right.$ $\left.\pi_{k}(h)\right]=0$, where $\left\|x_{i}-y_{i}\right\|<2^{-i+1} \epsilon$ and $\|h\|<\epsilon$.

Let $\beta$ be the flow generated by $\delta_{\alpha}-\operatorname{ad} i h$, where $\delta_{\alpha}$ is the generator of $\alpha$.

Let $A_{i}$ be the $\beta$-invariant $C^{*}$-subalgebra of $A$ generated by $y_{1}, \ldots, y_{i}$. Then $A_{i} \subset A_{i+1}$ and the union of $A_{i}$ is dense in $A$. Note that $E_{k j} \in \pi_{k}\left(A_{i}\right)^{\prime}$ for $j \geq \max \{k, i\}$. Since all $E_{k j}$ are of finite rank and a finite-dimensional covariant representation is a direct sum of finite-dimensional covariant irreducible representations, one can conclude that $\pi_{k} \mid A_{i}$ is a direct sum of finitedimensional covariant irreducible representations for all $k$, which in particular implies that $A_{i}$ is residually finite-dimensional.

When $\alpha$ is a flow on a $C^{*}$-algebra $A$ we denote by $F R(\alpha)$ the set of equivalence classes of finite-dimensional $\alpha$-covariant irreducible representations of $A$. Thus $\alpha$ is an RF flow if the intersection of all $\operatorname{Ker}(\pi), \pi \in F R(\alpha)$ is zero. If $\phi$ is an injection of $(A, \alpha)$ into $(B, \beta)$ we denote by $\phi^{\prime}(F R(\beta))$ the set of $\pi \in F R(\alpha)$ which is obtained as a sub-representation of $\rho \phi \mid A$ for some $\rho \in F R(\beta)$. Suppose that we are given an increasing sequence $\left(A_{n}, \alpha_{n}\right)$ of RF flows; we denote by $\phi_{m n}$ the embedding of $A_{m}$ into $A_{n}$ for $m<n$ intertwining $\alpha_{m}$ and $\alpha_{n}$. For each $m \in \mathrm{N}$ let $F R_{m}^{\prime}$ denote the intersection of all $\phi_{m n}^{\prime}\left(F R\left(\alpha_{n}\right)\right)$ with $n>m$. When the intersection of all $\operatorname{Ker}(\pi), \pi \in F R_{m}^{\prime}$ is zero for all $m$ we say that the increasing sequence $\left(A_{n}, \alpha_{n}\right)$ of RF flows is canonical.

Lemma 5.8. Let $\left(A_{n}, \alpha_{n}\right)$ be a canonical increasing sequence of RF flows and let $(A, \alpha)$ be the inductive limit of $\left(A_{n}, \alpha_{n}\right)$. There exists a family $S$ of $\alpha$-invariant pure states of $A$ such that if $\phi \in S$ then $\pi_{\phi} \mid A_{n}$ is equivalent to a direct sum of finite-dimensional covariant irreducible representations of $A_{n}$ for all $n \in \mathrm{N}$ and such that $\bigoplus_{\phi \in S} \pi_{\phi}$ is faithful. 
Proof. By using the notation before this lemma one finds, for any $m$ and $\pi \in F R_{m}^{\prime}$, a sequence $\left(\rho_{n}\right)_{n \geq m}$ such that $\rho_{n} \in F R\left(\alpha_{n}\right), \rho_{m}=\pi$, and $\rho_{n+1} \mid A_{n}$ contains $\rho_{n}$ as a subrepresentation. Fix a $\alpha_{m}$-invariant pure state $\phi_{m}$ of $A_{m}$ which induces $\rho_{m}$ as a GNS representation. One then finds a $\alpha_{m+1}$-invariant pure state $\phi_{m+1}$ of $A_{m+1}$ which induces $\rho_{m+1}$ and $\phi_{m+1} \mid A_{m}=\phi_{m}$. (Consider the embedding of $C=A_{m} / \operatorname{Ker} \rho_{m+1} \cap A_{m}$ into $D=A_{m+1} / \operatorname{Ker} \rho_{m+1} ; \phi_{m}$ is an $\alpha_{m}$-invariant pure state on a factor of the finite-dimensional $C^{*}$-algebra $C$. We pick up a factor $E$ of $D$ to which the factor of $C$ is mapped and then find an $\alpha_{m+1}$-invariant pure state $\phi_{m+1}$ of $E$, which we regard as a pure state on $A_{m+1}$.) By repeating this process we find a sequence $\left(\phi_{n}\right)_{n \geq m}$ such that $\phi_{n}$ is a $\alpha_{n}$-invariant pure state of $A_{n}$ which induces $\rho_{n}$ and $\phi_{n} \mid A_{n-1}=\phi_{n-1}$. Thus we can define a state $\phi$ of $A$ by $\phi \mid A_{n}=\phi_{n}$. One concludes that $\phi$ is an $\alpha$-invariant pure state. We denote by $U$ the unitary flow on $\mathscr{H}_{\pi}$ defined by $U_{t} \pi_{\phi}(x) \Omega_{\phi}=\pi_{\phi}\left(\alpha_{t}(x)\right) \Omega_{\phi}$. Note that $\mathscr{C}_{n}=\pi_{\phi}\left(A_{n}\right) \Omega_{\phi}$ is finite-dimensional and $U$-invariant. Since $\left(\mathscr{H}_{n}\right)$ is increasing and the union of all $\mathscr{H}_{n}$ is dense in $\mathscr{H}_{\phi}$ one concludes that $\pi_{\phi} \mid A_{n}$ is equivalent to a direct sum of covariant finitedimensional irreducible representations. Let $S$ denote the set of all $\phi$ for all the choices of $m, \pi \in F R_{m}^{\prime}$. Then the direct sum of $\pi_{\phi}$ is faithful on $A_{m}$ for any $m$ and thus it is faithful on $A$.

Proposition 5.9. Let $\alpha$ be a flow on a separable $C^{*}$-algebra. Then the following conditions are equivalent:

(1) There exists a faithful family of covariant irreducible representations of $(A, \alpha)$ which are quasi-diagonal.

(2) There exists an $\alpha$-cocycle $u$ and a canonical increasing sequence $\left(A_{n}, \alpha_{n}\right)$ of RF flows whose inductive limit is isomorphic to $(A, \mathrm{Ad} u \alpha)$.

Proof. Since $A$ is separable it follows from (1) that there is a countable family of covariant irreducible representations; $(1) \Rightarrow$ (2) follows from Lemma 5.7. The converse follows from Lemma 5.8.

Let $A$ be a unital separable simple quasi-diagonal $C^{*}$-algebra (e.g., a UHF algebra) and let $\alpha$ be an approximately inner flow on $A$ whose Connes spectrum is the whole $\mathrm{R}$. Then one can apply the above proposition to conclude that there is a $\alpha$-cocycle $u$ and a canonical increasing sequence $\left(A_{n}, \alpha_{n}\right)$ of RF flows whose inductive limit is isomorphic to $(A, \operatorname{Ad} u \alpha)$. This is because such a system has a covariant irreducible representation which induces a faithful representation of the crossed product (see [6]) and hence must be quasi-diagonal. 


\section{REFERENCES}

1. Blackadar, B., and Kirchberg, E., Generalized inductive limits of finite-dimensional $C^{*}$ algebras, Math. Ann. 307 (1997), 343-380.

2. Bratteli, O., and Kishimoto, A., AF flows and continuous symmetries, Rev. Math. Phys. 13 (2001), 1505-1528.

3. Bratteli, O., Kishimoto, A., Robinson, D. W. Approximately inner derivations, Math. Scand. 103 (2008), 141-160.

4. Bratteli, O., and Robinson, D. W., Operator Algebras and Quantum Statistical Mechanics I, Springer, New York 1979.

5. Choi, M-D., and Effros, E. G., The completely positive lifting problem for $C^{*}$-algebras, Ann. of Math. (2) 104 (1976), 585-609.

6. Kishimoto, A., Type I orbits in the pure states of a $C^{*}$-dynamical system II, Publ. Res. Inst. Math. Sci. 23 (1987), 517-526.

7. Kishimoto, A., Locally representable one-parameter automorphism groups of AF algebras and KMS states, Rep. Math. Phys. 45 (2000), 333-356.

8. Kishimoto, A., Approximately inner flows on separable $C^{*}$-algebras, Rev. Math. Phys. 14 (2002), 649-673.

9. Kishimoto, A., Multiplier cocycles of a flow on a $C^{*}$-algebra, J. Funct. Anal. 235 (2006), 271-296.

10. Kishimoto, A., Robinson, D. W., On unbounded derivations commuting with a compact group of $*$-automorphisms, Publ. Res. Inst. Math. Sci. 18 (1982), 1121-1136.

11. Kishimoto, A., Robinson, D. W., Quasi-diagonal flows, J. Operator Theory 66 (2011), $353-$ 384.

12. Lin, H., Almost commuting self-adjoint matrices and applications, pp. 193-233 in: Operator Algebras and Their Applications, Proc. Waterloo, Ont. 1994/95, Fields Inst. Commun. 13, Amer. Math. Soc., Providence, RI 1997.

13. Pisier, G., Factorization of Linear Operators and Geometry of Banach Spaces, CBMS Regional Conference Series in Math. 60, Amer. Math. Soc., Providence, RI 1986.

14. Sakai, S., Operator Algebras in Dynamical Systems, Encycl. Math. Appl. 41, Cambridge Univ. Press, Cambridge 1991.

15. Voiculescu, D., A note on quasi-diagonal $C^{*}$-algebras and homotopy, Duke Math. J. 62 (1991), 267-271.

16. Voiculescu, D., Around quasidiagonal operators, Integral Equations Operator Theory 17 (1993), 137-149.

DEPARTMENT OF MATHEMATICS

HOKKAIDO UNIVERSITY

SAPPORO

JAPAN

E-mail: kishi@math.sci.hokudai.ac.jp 